\title{
MISR-GOES 3D Winds: Implications for Future LEO-GEO and LEO-LEO Winds
}

\author{
James L. Carr ${ }^{1, *}$, Dong L. Wu ${ }^{2}$, Michael A. Kelly ${ }^{3}$ and Jie Gong ${ }^{4}$ \\ Carr Astronautics, 6404 Ivy Lane, Suite 333, Greenbelt, MD 20770, USA \\ NASA Goddard Space Flight Center, Greenbelt, MD 20770, USA; dong.l.wu@nasa.gov \\ Johns Hopkins Applied Physics Laboratory, Laurel, MD 20723, USA; michael.kelly@jhuapl.edu \\ 4 NASA Goddard Space Flight Center, Greenbelt, MD 20770, USA; jie.gong@nasa.gov \\ * Correspondence: jcarr@carrastro.com; Tel.: +1-301-220-7350
}

Received: 4 October 2018; Accepted: 21 November 2018; Published: 27 November 2018

check for updates

\begin{abstract}
Global wind observations are fundamental for studying weather and climate dynamics and for operational forecasting. Most wind measurements come from atmospheric motion vectors (AMVs) by tracking the displacement of cloud or water vapor features. These AMVs generally rely on thermal infrared (IR) techniques for their height assignments, which are subject to large uncertainties in the presence of weak or reversed vertical temperature gradients near the planetary boundary layer (PBL) and tropopause folds. Stereo imaging can overcome the height assignment problem using geometric parallax for feature height determination. In this study we develop a stereo 3D-Wind algorithm to simultaneously retrieve AMV and height from geostationary (GEO) and low Earth orbit (LEO) satellite imagery and apply it to collocated Geostationary Operational Environmental Satellite (GOES) and Multi-angle Imaging SpectroRadiometer (MISR) imagery. The new algorithm improves AMV and height relative to products from GOES or MISR alone, with an estimated accuracy of $<0.5 \mathrm{~m} / \mathrm{s}$ in AMV and $<200 \mathrm{~m}$ in height with $2.2 \mathrm{~km}$ sampling. The algorithm can be generalized to other LEO-GEO or LEO-LEO combinations for greater spatiotemporal coverage. The technique demonstrated with MISR and GOES has important implications for future high-quality AMV observations, for which a low-cost constellation of CubeSats can play a vital role.
\end{abstract}

Keywords: 3D-Winds; atmospheric motion vectors (AMVs); MISR; GOES-R; planetary boundary layer (PBL); stereo imaging; parallax; CubeSats

\section{Introduction}

Atmospheric motion vectors (AMVs) derived by tracking cloud and water vapor features in satellite imagery have been one of the key observations used in numerical weather prediction (NWP) systems [1,2]. The impact of AMVs on forecasts is the greatest in the tropics, Southern Hemisphere, and regions where data are otherwise sparse. In addition to the tropics, Gelaro and Zhu [3] identified other regions where forecast error is most sensitive in observing system experiments (OSEs). These regions are often associated with strong atmospheric energetics and baroclinic instability where radiosonde data are sparse and NWP must depend heavily on satellite AMVs. One of the main uncertainties associated with the AMV measurements is the height assignment, which can prevent AMVs from being assimilated into NWP systems, especially in the presence of a strong vertical wind shear and complex thermal structures (e.g., tropopause folding, boundary layer inversion). Accuracy of height assignment was assessed in a number of studies by comparing Moderate Resolution Imaging Spectroradiometer (MODIS) and geostationary satellite cloud top heights (CTHs) with CTHs from the Cloud-Aerosol Lidar with Orthogonal Polarization (CALIOP) instrument and other independent measurements [4-7]. 
The AMV technique has several key assumptions and limitations regarding tracked atmospheric features (e.g., clouds, water vapor) and the interpretation of their evolution as winds. Fundamentally, features are assumed to be traveling together with the wind so that feature motion is equal to air motion and therefore such features are assumed to be passive tracers [8]. This assumption can break down in the case of standing waves, such as orographic waves, where clouds anchor on the top of mountains. Feature patterns that are tracked must be distinctive, neither uniform nor periodic, so that they can be uniquely identified from one location to another. A uniform feature will lead to no solution in pattern matching whereas a wavy pattern can lead to multiple solutions. To be considered the same feature, no major distortion in feature patterns should occur over consecutive images. Clouds may change shape or grow over the period of consecutive images but must not change shape so much as to be unrecognizable as the previous pattern. This requirement may be limited by latitude-dependent cloud spatiotemporal variability. Generally, vertical motion is neglected; although atmospheric features can grow vertically during a several-minute tracking interval, the resulting motion from tracking features in consecutive images is assumed to be due to horizontal motion only. Finally, each AMV is generally assigned one height in the atmosphere that would represent where the pattern becomes optically thick. The assigned height can be considered the cloud top if tracking a cloud pattern. In the case of multiple cloud layers and particularly when the top layer is semitransparent as with cirrus clouds, the pattern matcher picks the layer offering the strongest contrast as the pattern height.

In this paper, we show the benefit of data fusion from geostationary (GEO) and low-earth orbit (LEO) satellites for producing high quality three-dimensional (3D) AMVs with a multi-platform, multi-angle stereoscopic technique. We use the term "3D Winds" primarily to signify the positioning of horizontal AMVs three-dimensionally in the atmosphere, but we also examine relaxation of the horizontal constraint in our retrieval algorithm. We demonstrate the benefits of our 3D-Winds algorithm using data from the Multi-angle Imaging SpectroRadiometer (MISR) instrument and the new Geostationary Operational Environmental Satellite series-R (GOES-R) satellites over the Contiguous United States (CONUS). The AMVs jointly retrieved from MISR and GOES-16/17 show significant improvements in AMV and height measurement quality relative to AMVs from GOES or MISR alone. The results from this study highlight the potential of synergistic observations between advanced GEO imagers and a LEO constellation of CubeSat multi-angle sensors for future 3D global wind observations.

\section{AMVs and Height Assignments}

The primary infrared (IR) methods used for AMV height assignment are the IR window [9], $\mathrm{CO}_{2}$ slicing [10,11], and $\mathrm{H}_{2} \mathrm{O}$ intercept [12,13] techniques. One approach to the IR-window method assumes that the mean of the lowest $20 \%$ (coldest) brightness temperature values in the target sample is the temperature at the cloud top. This temperature is compared to a numerical forecast of the vertical temperature profile to determine the cloud height. The cross-correlation contribution (CCC) method is a variation on the IR-window method. CCC assigns the height using temperatures of pixels ranked according the importance of their contribution to the pattern-matching solution [14]. In the case of opaque clouds, the sensed temperature used by the IR-window method will be representative of the modeled temperature at the pressure level (or height) of the tracked cloud top; however, the IR-window method is known to be challenged in the presence of semitransparent clouds for which this is not necessarily true [15]. The $\mathrm{CO}_{2}$-slicing method is intended to work well for both opaque and semitransparent clouds. Cloudy and clear radiance differences in one or more carbon dioxide bands (e.g., 13.3, 13.6, 13.9, or $14.2 \mu \mathrm{m}$ on MODIS) and infrared window bands are ratioed and compared to the theoretical ratio of the same quantities for a range of cloud pressures. The cloud pressure that gives the best match between the observed and theoretical ratios is chosen. Zhang and Menzel [16] proposed an improved $\mathrm{CO}_{2}$-slicing method by taking into account cloud emissivity. The $\mathrm{H}_{2} \mathrm{O}$-intercept method of height determination can be used as an additional metric or in the absence of a $\mathrm{CO}_{2}$ band. This method examines the relationship between clusters of clear and cloudy pixel values in water vapor-infrared window brightness temperature space, predicated on the fact that radiances from a 
single cloud deck for two spectral bands vary linearly with cloud fraction within a pixel. The line connecting the clusters is compared to theoretical calculations of the radiances for different cloud pressures. The intersection of the two gives the cloud height. The $\mathrm{H}_{2} \mathrm{O}$-intercept and $\mathrm{CO}_{2}$-slicing methods have challenges when applied to low-level or multilayered clouds. Problems occur when the clear-cloudy radiance difference is too small. Cloud pressures greater than $700 \mathrm{hPa}$ (lower in altitude) are generally not retrievable with either of these methods. Velden et al. [17] found that AMVs at coarser vertical resolutions, or layer-averaged values, were correlated better with radiosonde data than those assigned at discrete levels.

In current and previously operational GEO satellites (e.g., GOES and METEOSAT), AMVs are produced from visible (VIS), infrared (IR) and water vapor (WV) channel imagery. Typical height distributions of AMV occurrence frequency are illustrated in Figure 1 from METEOSAT-10 observations in August 2015. AMVs are basically available in a wide range of pressure levels between $60^{\circ} \mathrm{S}-60^{\circ} \mathrm{N}$. It is common in these GEO AMV observations that they are sparse at low heights, or high-pressure levels, due to poor quality statistics prior to quality control.
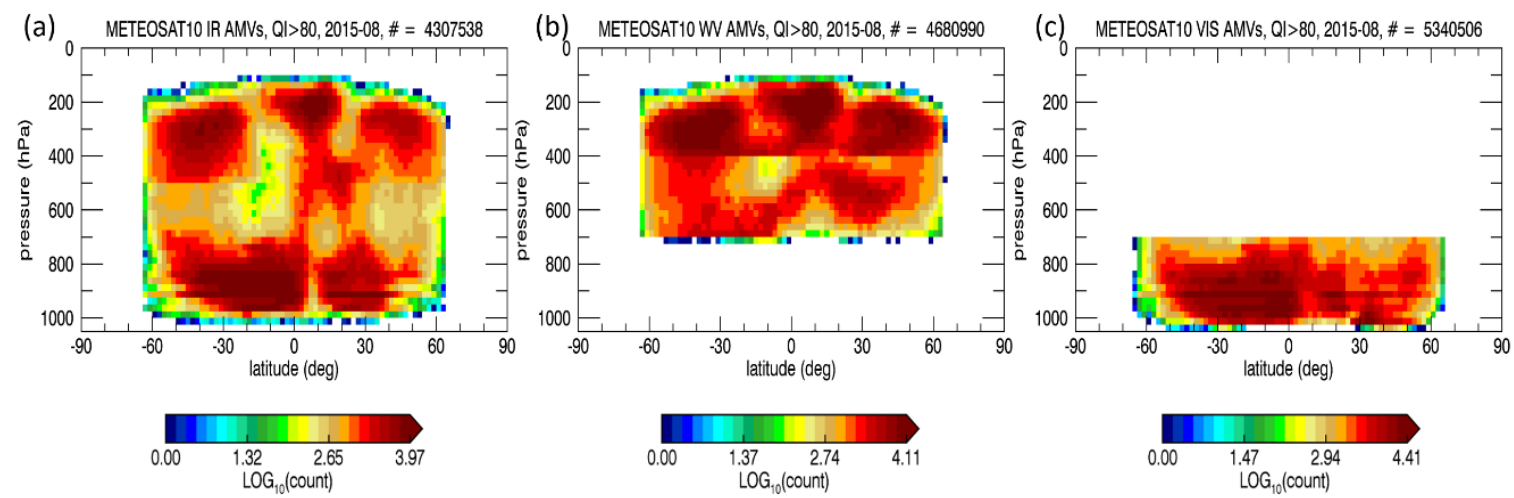

Figure 1. Vertical and latitudinal distributions of METEOSAT-10 AMVs derived from (a) IR, (b) WV, and (c) VIS channels. The three methods have an overlapping vertical coverage, with the IR winds in the entire troposphere, WV winds mostly in the upper troposphere and the VIS winds in the lower troposphere (Figure courtesy of Á. Horváth, University of Hamburg).

The earlier generation of operational GEO environmental satellites is represented by GOES 8-15 and the Meteosat Second Generation (MSG) satellites, METEOSAT 8-11. The first imagers in this series of GOES satellites have nadir detector footprints of $1 \mathrm{~km}, 4 \mathrm{~km}$ and $8 \mathrm{~km}$ for VIS, IR and WV channels respectively. Later imagers in the GOES series replaced existing 6.7 and $12 \mu \mathrm{m}$ channels with 6.5 and $13.3 \mu \mathrm{m}$ channels and improved spatial resolution to $4 \mathrm{~km}$ for all IR and WV channels. These legacy GOES imagers cover the full disk in approximately $30 \mathrm{~min}$, but can cover and therefore update CONUS, North and South American scenes more frequently. MSG covers the full-disk every $15 \mathrm{~min}$ with a spatial resolution of $1 \mathrm{~km}$ in a high-resolution VIS channel and $3 \mathrm{~km}$ for 11 other channels. The advanced imagers on GOES-16 (2016-present) and Himawari-8 (2015-present) represent the new generation of geostationary imaging systems, which can refresh coverage of the full-disk every 15 , 10 , or $5 \mathrm{~min}$ in 16 spectral channels and with improved nadir resolution $(0.5 \mathrm{~km}, 1 \mathrm{~km}$ and $2 \mathrm{~km})$. The CONUS, which is well covered by GOES-16, is refreshed every $5 \mathrm{~min}$ and smaller mesoscale (MESO) scenes can be refreshed with a 1-min or 30-s cadence. The improved spatiotemporal sampling of these new generation GEO systems allows more features to be tracked for AMVs, yielding many more AMVs in the same region over the earlier generation of GEO satellites [18].

Satellite stereo-photogrammetry is a promising technique for deriving AMVs with an alternative height assignment method, and the technique has been used since the beginning of the space era [19-25]. Since the pattern matching for stereo height uses the same matching algorithm used for AMV determination, the height from the stereo method should be most representative of the pattern height. By imaging cloud features from multiple view angles within a short time interval, 
the stereo technique can overcome many of the difficulties associated with IR height assignment methods. For example, the height determination from the stereo technique is insensitive to atmospheric temperatures and is therefore not limited by atmospheric thermal structures or complex surface types as with thermal IR techniques. However, the height assignment from stereo-photogrammetry is sensitive to image registration errors. Improvements in registration accuracy have been made with the new generation of GEO imagers represented by GOES-16 and Himawari-8, which assures that stereo-photogrammetry will be a valuable technique.

The MISR instrument (2000-present) offers nine viewing angles (Nadir, $\pm 26^{\circ}, \pm 46^{\circ}, \pm 60^{\circ}, \pm 70^{\circ}$ ), each designated by a letter (An, Aa, Af, Ba, Bf, Ca, Cf, Da, Df), in four spectral bands (446, 558, $672,866 \mathrm{~nm}$ ) and with global coverage in swaths of $\sim 350 \mathrm{~km}$ [26-29]. LEO stereo-photogrammetry using MISR produces winds and cloud heights from disparities (i.e., apparent relative shifts of features) between camera pairs, specifically Bf-to-An, Bf-to-Df, Ba-to-An and Ba-to-Da. MISR measures wind and cloud heights using all the disparities (i.e., apparent relative shifts of features) from its nine angular views. The disparities from the oblique angles, benefiting from the curvature of Earth's surface along track, break the degeneracy between along-track wind and cloud height in MISR wind retrievals [27]. However, the independence of the two retrieved quantities is not perfect, which produces correlated errors in the along-track wind and its height assignment $[26,28]$. The retrieved height error is proportional to the along-track wind error by a factor of 75-93 $\mathrm{m}$ for every $1 \mathrm{~m} / \mathrm{s}$ [30].

Adding independent views from another satellite also helps to remove the degeneracy between along-track wind and height, which is inherent in multi-angle observations from a single LEO platform, but also introduces problems of working with data from systems that have different characteristics and spatial resolutions. This paper shows that such problems can be solved in our newly-developed 3D-Wind algorithm. Here we demonstrate joint AMV and height retrieval with collocated MISR and GOES imagery, to show that the resulting products are more accurate than those derived from either MISR or GOES alone. This LEO-GEO matching has become attractive in light of the advanced high-resolution imaging sensors and high-accuracy pointing from GEO as represented by GOES-16 and Himawari-8. This study is the first attempt to use high-quality LEO-GEO retrievals to characterize the quality of current LEO and GEO AMV products.

\section{Multi-Angle and Multi-Platform Methods and Results}

Observation of the parallax of a feature when observed from two different vantage points (or stereo) provides direct information about its height independent of atmospheric models and thermal structures. The stereo technique has advantages over the passive thermal IR approach in determining a feature's height. It avoids any dependence on atmospheric thermal structure, and thus avoids the potential for large errors in height assignment at mid-to-high latitudes or in the boundary layer where the temperature lapse rate is small or reversed (e.g., [5,7]).

We first discuss GEO-GEO combinations considering the GOES constellation and then explore LEO-GEO combinations with MISR and GOES in some detail in the following subsections. LEO-LEO combinations can also be used to perform stereo imaging. For example, MODIS on Terra and Aqua can obtain a triplet of imagery in $\sim 1.5 \mathrm{~h}$ to produce AMV measurements at latitudes poleward of $70^{\circ}$. There is an existing MODIS-MODIS polar AMV algorithm that is fully automated, and the product, available since September 2002, has been used operationally for weather forecasting [31]. However, the algorithm uses a priori height assignments to correct for parallax to improve wind vectors instead of using the parallax to solve for height. This polar AMV dataset has been especially valuable because it provides coverage outside the range of coverage by the GEO constellation. There is a $\sim 10^{\circ}$ gap in latitude coverage between GEO and polar AMVs, since the spatial coverage is generally equatorward of $60^{\circ}$ latitude for GEO satellites and poleward of $70^{\circ}$ latitude for the polar satellites. Within this latitudinal gap, the dynamically active polar jet stream can often be found, and the lack of observations can lead to poor model initialization and rapidly growing errors in the forecasts. Research 
has shown that the addition of the polar winds can be especially important in the active polar jet stream region [32,33]. To fill this gap, a GEO-LEO algorithm was developed, which requires an advanced image compositing technique to blend the data from a variety of satellites: GEO (GOES, Meteosat, MTSAT) and LEO satellites (NOAA-15 through NOAA-19 and Metop-A; NASA's Terra and Aqua). The GEO-LEO AMVs can be produced at the best spatial resolution from composite images of different satellites that vary in sampling time and viewing angle [34]. These satellite image composites are being routinely generated every 15 min for infrared window channels at $4 \mathrm{~km}$ resolution in polar stereographic projection over each pole. Like other AMVs, the GEO-LEO AMVs are derived from tracking a triplet of images separated by 30-45 min from these composites.

Other sensor combinations can be exploited to augment the polar 3D-Wind capability as well, including MODIS with the Atmospheric Infrared Sounder (AIRS) [35] and paired Advanced Very High Resolution Radiometer (AVHRR) instruments on the Metop series [36].

\subsection{GEO-GEO Multi-Platform Winds}

The idea of using stereo imaging to observe clouds in 3D where there is simultaneous and overlapping coverage by two GEO spacecraft was described by Hasler [21]. We continued with this concept to make quantitative retrievals of $\mathrm{CTH}$ from the legacy GOES constellation as an internal research and development (IR\&D) project at Carr Astronautics [37] using archival imagery from GOES-13, -14, and -15 of Hurricane Sandy. The motivation for our work was to demonstrate that a stereo CTH product could be sufficiently accurate to be useful as an operational product, particularly with the advanced capabilities expected from GOES-R. At the time of Sandy, GOES-13 and -15 were being operated as the eastern and western satellites, respectively, and GOES-14, in a storage orbit over a central longitude, was taken out and put in operation for a test campaign. This configuration provided coverage across much of the northern hemisphere by two satellites and much of the CONUS by three satellites. We developed a linear cloud height retrieval algorithm that derived CTH from single or paired parallax measurements made by matching templates from GOES-14 against GOES-13 and -15 imagery.

The key enabling parameters for high-quality stereo-CTH retrievals from two GEO satellites are the spatial resolution and Image Navigation and Registration (INR), which permit accurate positioning of features with respect to absolute geographic coordinates. The results using the GOES visible channel across the northern hemisphere are promising after applying corrections for some INR-related systematic errors so that the measured height over clear-sky terrain matched a terrain model. This provided a necessary validation of the INR performance over the legacy GOES constellation [38], for which both INR and spatial resolution are improved in the GOES-R series [39]. We compared the CTH retrievals from GOES stereo imaging against CALIOP laser altimetry [40] when CALIOP traversed nearly coincident GOES scenes (Figure 2).

The stereo method for CTH retrievals was further extended to include tracking pattern motions between consecutive images using the same pattern-matching algorithm. We modeled pixel times and simultaneously solved for both AMVs and height from the observed disparities in the cloud patterns. The new capability yielded first CONUS and later full-disk 3D-Wind products [41]. This version is the predecessor of the 3D-Winds algorithm that we will discuss in depth in Section 3.2. Figure 3 shows an example of a two-satellite full-disk product using GOES-13 $\left(75^{\circ} \mathrm{W}\right)$ and GOES-16 when it was positioned at $89.5^{\circ} \mathrm{W}$ for testing. A large part of the full disk is covered in their overlap since the two satellites are only $15^{\circ}$ apart. For two GEO AMVs, there always is a tradeoff between the accuracy of height retrievals and breadth of coverage. 


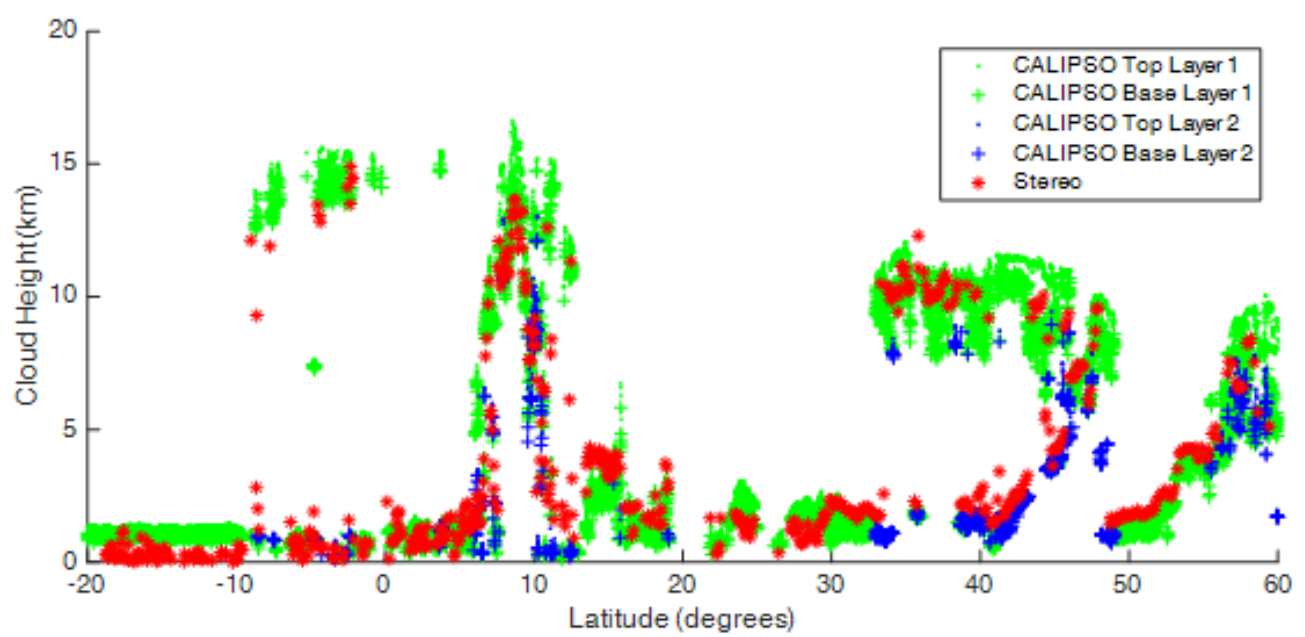

Figure 2. CALIOP and Stereo CTH Comparison for 19:45Z, 24 October 2012. The comparison is limited to the orbit path where the Cloud-Aerosol Lidar and Infrared Pathfinder Satellite Observation (CALIPSO) satellite passed across the stereo CTH product at nearly the same time. A total of 280 stereo-CTH retrievals can be associated with CALIOP layer 1 (i.e., $>$ bottom and $<$ top $+4 \mathrm{~km}$ ), with a population mean height difference of $-2 \mathrm{~m}$ and a standard deviaiton of $784 \mathrm{~m}$ (Figure courtesy of Houria Madani, Carr Astronautics).

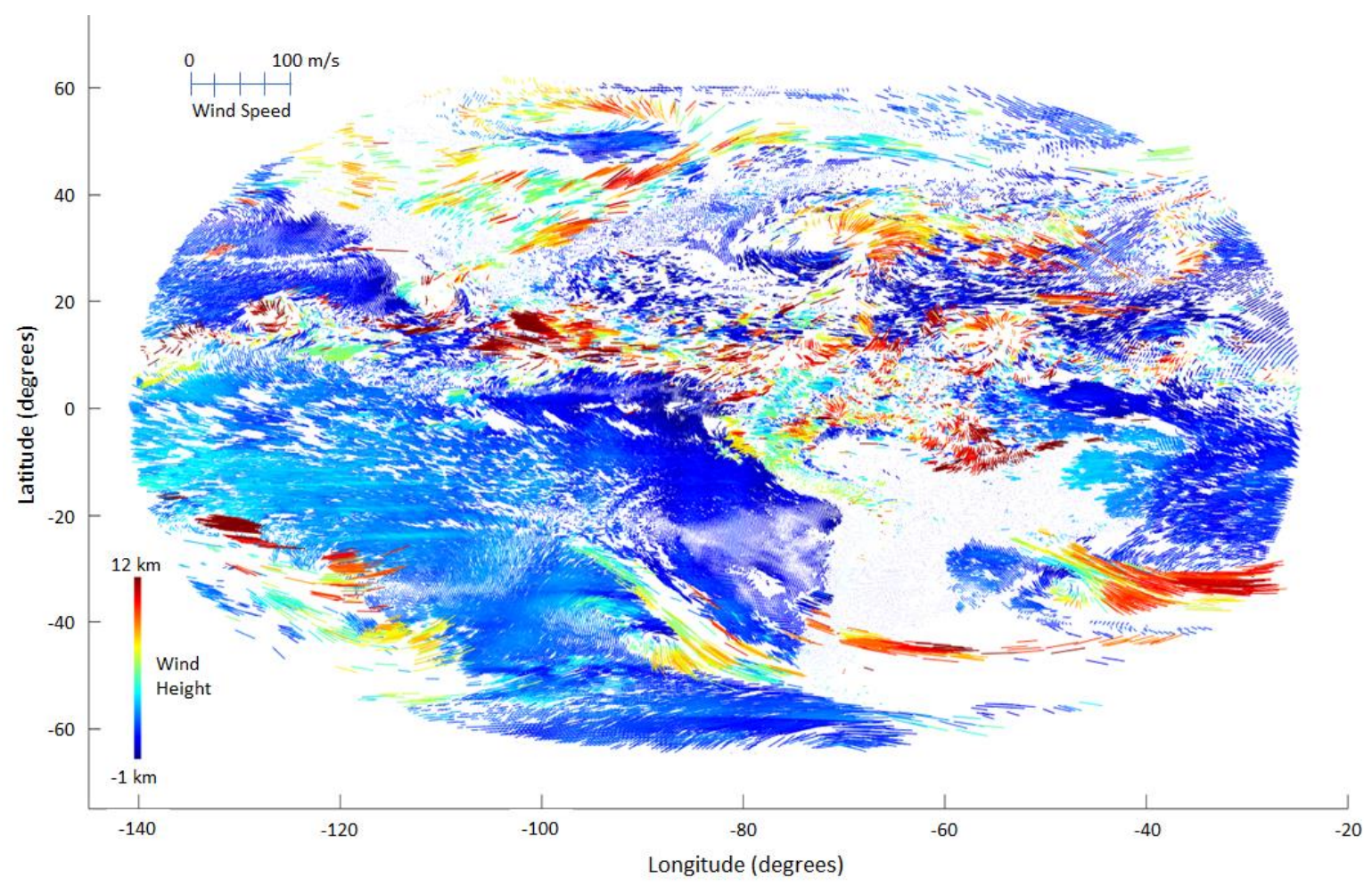

Figure 3. Full Disk 3D Wind Field from GOES-13 (East) and -16 (Test Slot) for 18:00Z, 17 September 2017.

\subsection{LEO-GEO Multi-Platform Winds}

In this study we extend the 3D-Wind algorithm from GEO-GEO to LEO-GEO pairs and apply it to recent MISR and GOES-16/17 observations. The detailed mathematical model of the MISR-GOES 3D-Wind retrieval algorithm can be found in Appendix A. The algorithm generates LEO-GEO retrievals one block at a time from the MISR red channel and GOES Advanced Baseline Imager (ABI) Band 2 (B02), the closest spectral match to MISR. Each block consists of a subset of a MISR orbital path that 
measures 512 pixels along-track by 2048 pixels across track. We have restricted our attention to passes over CONUS where the ABI is typically executing its Mode-3 timeline. Mode 3 offers continuous coverage of CONUS on a 5-min refresh cycle. Figure 4a shows a typical overpass with the outline of the MISR blocks projected over an approximately simultaneous GOES-16 B02 CONUS scene. In this case, block numbers 53 to 77 of the MISR product are fully contained within the CONUS product with appropriate margin to allow for feature matching near the CONUS boundaries. We always process a block together with its successor and predecessor to eliminate boundary effects between MISR blocks. The MISR blocks 53 through 77 shown in Figure 4a belong to path 24 and orbit 98,797 of the Terra satellite, and so we designate the dataset using the shorthand notation "P024O098787B53:77" for MISR Path, Orbit and Block numbers. Figure $4 \mathrm{~b}$ is the 3D-wind field for P024O098787B53:77, with AMVs represented as line segments scaled in proportion to the wind speed and color-code by height above the WGS84 ellipsoid. It is a simple matter to convert to height above mean sea level but to state the height as a pressure level requires help from an atmospheric model.

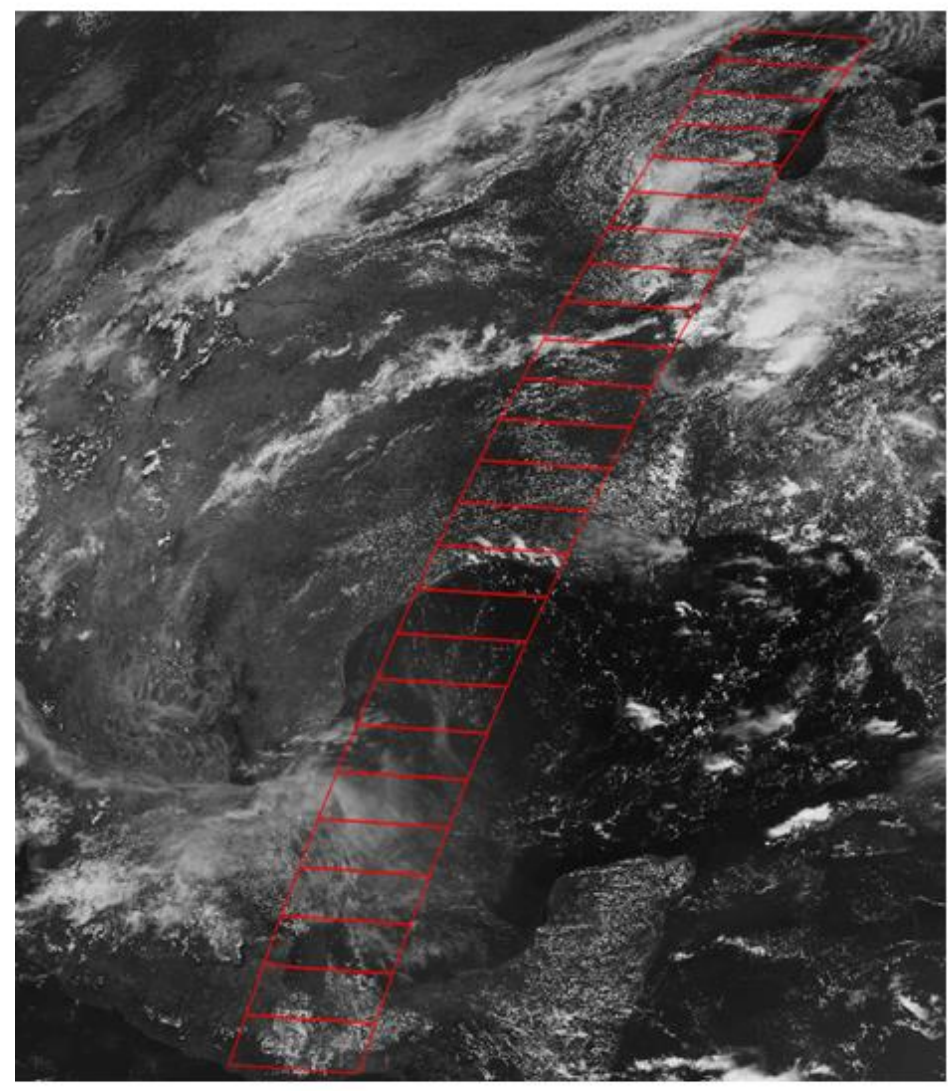

(a) MISR P024

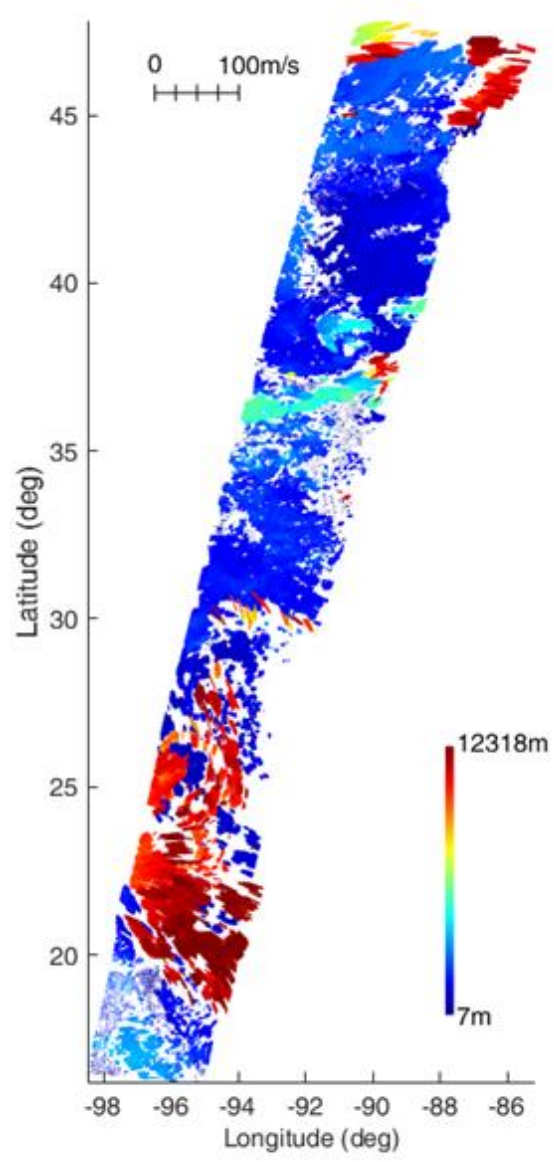

(b) 3D Winds

Figure 4. (a) MISR Blocks over CONUS. Terra orbital Path 24 on Orbit number 98,787 is displayed over a GOES-16 B02 CONUS scene from 15 July 2018, day 196 of 2018, near 17:00Z (approximately satellite noon) and close in time to the overpass. (b) Retrieved 3D-Wind field for P024O098787B53:77 over CONUS as shown in Figure 4a. Clouds are distributed from the boundary layer to near the tropopause along this path.

\subsubsection{D-Wind Algorithm}

MISR GRP_ELLIPSOID radiances, which are rectified to the WGS-84 ellipsoid, are used in the 3D-Wind retrieval. GOES ABI Level-1b products are similarly referenced to the ellipsoid in accordance with the fixed-grid definition for ABI products [42]. The lines-of-sight to an elevated object from 
the different cameras of MISR and the ABI when projected onto the ellipsoid, as is the case with ellipsoid-rectified imagery, are assigned different geographic coordinates. Therefore, an object will appear to be displaced relative to a reference camera. In general, this apparent displacement or disparity, can be attributed to motion of the object during the time between looks, 3D parallax, or a combination thereof. This is the foundation of the approach to operational MISR cloud heights and winds [43]. Here, we designate the MISR nadir-looking (An) camera to be our reference and pattern match to the other MISR cameras and three repetitions of an ABI CONUS scene. MISR brings excellent observability of cloud height with some mixing between along-track winds and height, and GOES brings excellent observability of cloud motion; together, the full 3D geometry of clouds and their motion are well observed.

The MISR GRP_ELLIPSOID imagery is rendered over the Space Oblique Mercator (SOM) projection [44]. Before attempting pattern matching with ABI, we first remap a relevant subset of ABI imagery from its native ABI fixed grid into the MISR SOM projection with the resolution of the MISR red band. ABI B02 imagery has a nadir resolution of $500 \mathrm{~m}$ in comparison with the $275 \mathrm{~m}$ resolution of the MISR red band, which means that patterns appear somewhat blurry in the remapped ABI imagery as compared to the MISR red band; however, they can still be matched. We are using an optimized Normalized Cross-Correlation (NCC) algorithm [45] to identify matches and measure disparities. Subpixel resolution is achieved by modeling the correlation surface in the neighborhood of its maximum as a quadratic surface and interpolating [46]. We cover each $512 \times 2048$ block of An-camera imagery with a uniform mesh using an 8-pixel sampling $(2.2 \mathrm{~km})$. Templates of $40 \times$ 40 pixels are matched to measure disparities. This simple approach provides ample matches from which to retrieve 111,894 3D-Wind vectors in Figure 4b, although more sophisticated means of feature selection, template sizing, and matching may have advantages and potentially measure atmospheric motion at different spatial scales.

The algorithm nominally measures disparities from the MISR aft- and forward-looking cameras to nadir (Aa-to-An, Af-to-An), and between each of a triplet of sequential GOES CONUS scenes and An for which the middle of the GOES temporal triplet is as close to a simultaneous match to An as possible, but it is possible to match with the MISR B, C, and D cameras as well. Modeling the time associated with each template and its match is critical to a successful 3D-Wind retrieval. Since we work with resampled products, times for pixels have only an approximate meaning. The centers of MISR blocks are time tagged within the MISR GRP_ELLIPSOID product and we can therefore deduce their approximate pixel times by assuming a nearly linear advance of time between block centers along the ground track. Assigning times to GOES pixels is slightly more complicated and requires detailed knowledge of the operation of ABI as it follows its timeline. ABI L1b product times always reference the start of each scene for B02. We know the pattern of coverage by swaths and the release of fragments from the pipelined resampling process within the ABI Ground System [47] and can therefore generally assign a swath number to each product row with some confidence. This allows for reliable time assignments except near swath seams where there is in general some ambiguity about which swath a product pixel belongs. Despite this complication, a substantial majority of GOES product pixels can be time tagged with an accuracy that is adequate for LEO-GEO AMV retrievals as we will show. Since there is no synchronization between MISR and GOES, the An-camera block can be out of phase with the GOES scene nearest in time by as much as $\pm \frac{1}{2}$ of the GOES refresh period and will vary from one block to the next.

A geometric retrieval is the final step in the 3D-Winds algorithm. Each template yielding a match across the intended subset of MISR cameras and the GOES triplet qualifies as a site for a retrieval. A prescreening is performed for data quality before the retrieval and screening after retrieval to verify that the model adequately explains the set of measured disparities at each site. The first prescreening is an autocorrelation test of the template being matched with the An-camera image from which it came. This test helps to eliminate featureless areas such as those over water under clear skies where an autocorrelation can show a large nonsensical disparity. The next screening is performed on 
peak correlation coefficient relative to threshold values. In rare cases, the NCC correlation surface interpolation algorithm may fail because the modeled quadratic surface is a saddle surface and such cases are screened out as interpolation is impossible. Finally, a consistency check is carried out for the disparities between those measured with forward and aft-looking camera pairs and between forward and backward temporal differences in the GOES imagery. The prescreening in the 3D-Wind algorithm filters out most disparities for which no sensible interpretation can be derived and enables statistical outlier testing on the residuals after retrieval to be effective in removing the rest. Figure 5 shows the 3D-Wind product over a single block at the sites where a successful match has been made between An and the two other A cameras and An and a triplet of GOES-16 images. Figure 6 shows the disparities that were measured by pattern matching and used in the retrievals for AMVs and their heights.

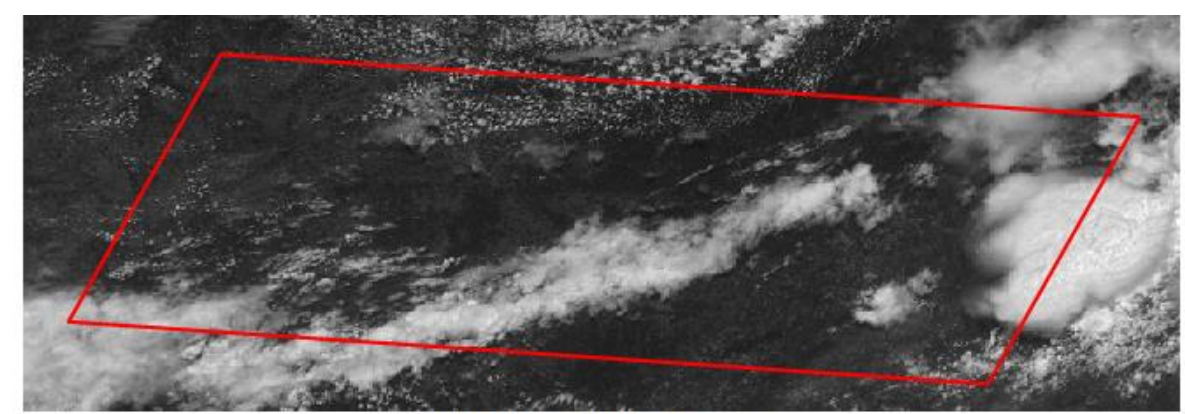

(a) MISR P024O098787B61

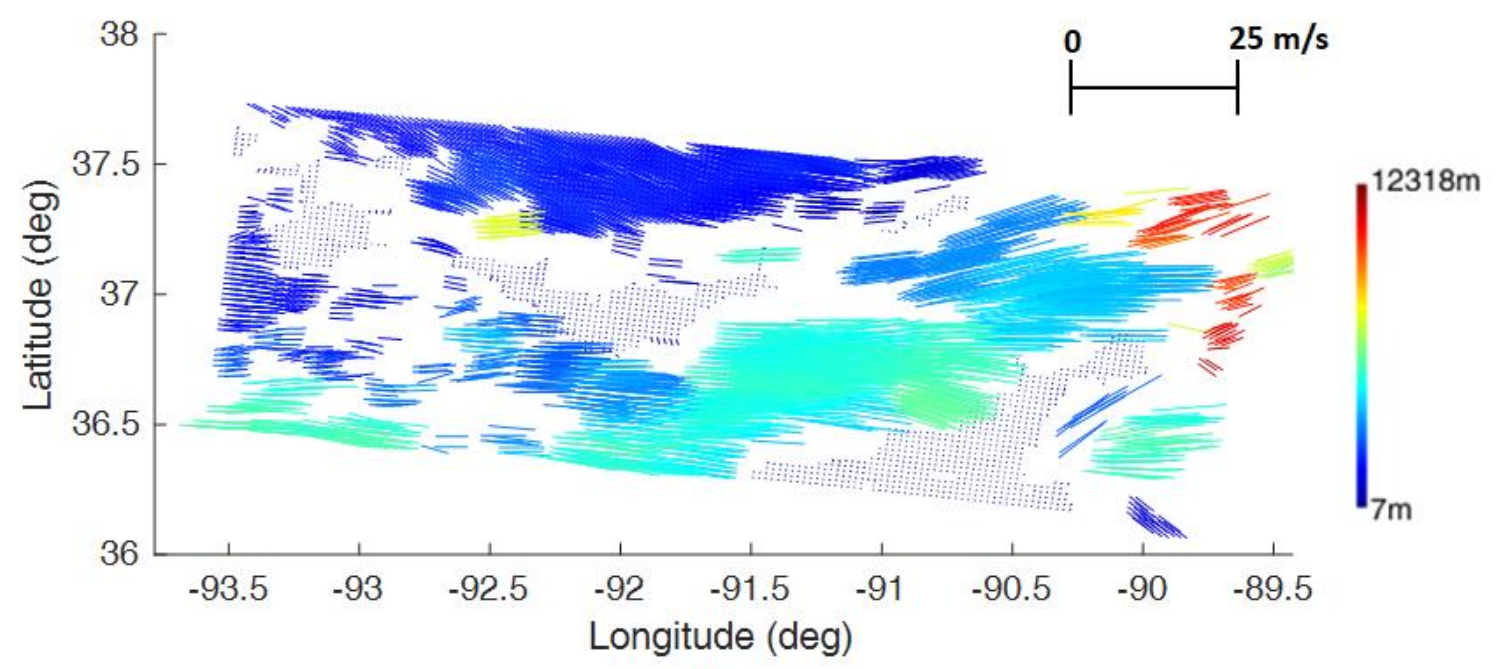

(a) 3DWinds

Figure 5. Retrieved 3D winds and heights over a single MISR block: (a) MISR block over GOES-16 Band 02 imagery from a nearly simultaneous CONUS scene (north up and east right) with a mixture of different cloud types and clear sky, (b) Retrieved AMVs and height assignments.

Coupled AMV and height retrieval is an optimization process that fits the disparities at each site with a state model consisting of three position corrections and two AMVs defined parallel to the tangent plane to the ellipsoid at each site and optionally a third AMV parallel to the local vertical. The two components of the position correction in the tangent plane include the correction to the MISR coordinates due to parallax and the remaining state is the pattern height above the ellipsoid. The algorithm finds the weighted least-squares solution that minimizes the residual disparities relative to the model by iteratively refining the state solution until convergence is achieved to the desired tolerance. Weighting of residuals is inversely proportional to the respective resolutions squared and accords greater weight to MISR in the solution. The solution is fully nonlinear, which is important for 
high clouds and oblique looks where the first iteration (linear) solution can deviate from the converged (nonlinear) solution by up to $100 \mathrm{~m}$ vertically. It is useful to include bundle adjustment states that apply globally to all sites and compensate for small systematic errors in the alignment between GOES and MISR imagery block by block, as geo-registration errors can be significant $[48,49]$. We have only attempted a zeroth order bundle adjustment to represent a correction applied in the tangent plane at each site that translates the MISR imagery for all cameras with respect to GOES in each of the cardinal directions, roughly a correction in roll and pitch (a higher-order adjustment could compensate for rotation and skew distortions such as yaw). Therefore, there are $(2+1+2) \times N+2$ states over $N$ sites, with two position parallax corrections, one height, and two velocity states per site and two bundle-adjustment states. Each of the five states at the $N$ sites only affect the disparities at their own site, while the bundle adjustment acts globally across all sites. We use sparse matrix methods to solve the optimization problem, which is quite efficient even with $N \sim 10^{4}$. The math model for the 3D-Wind algorithm is found in Appendix A.

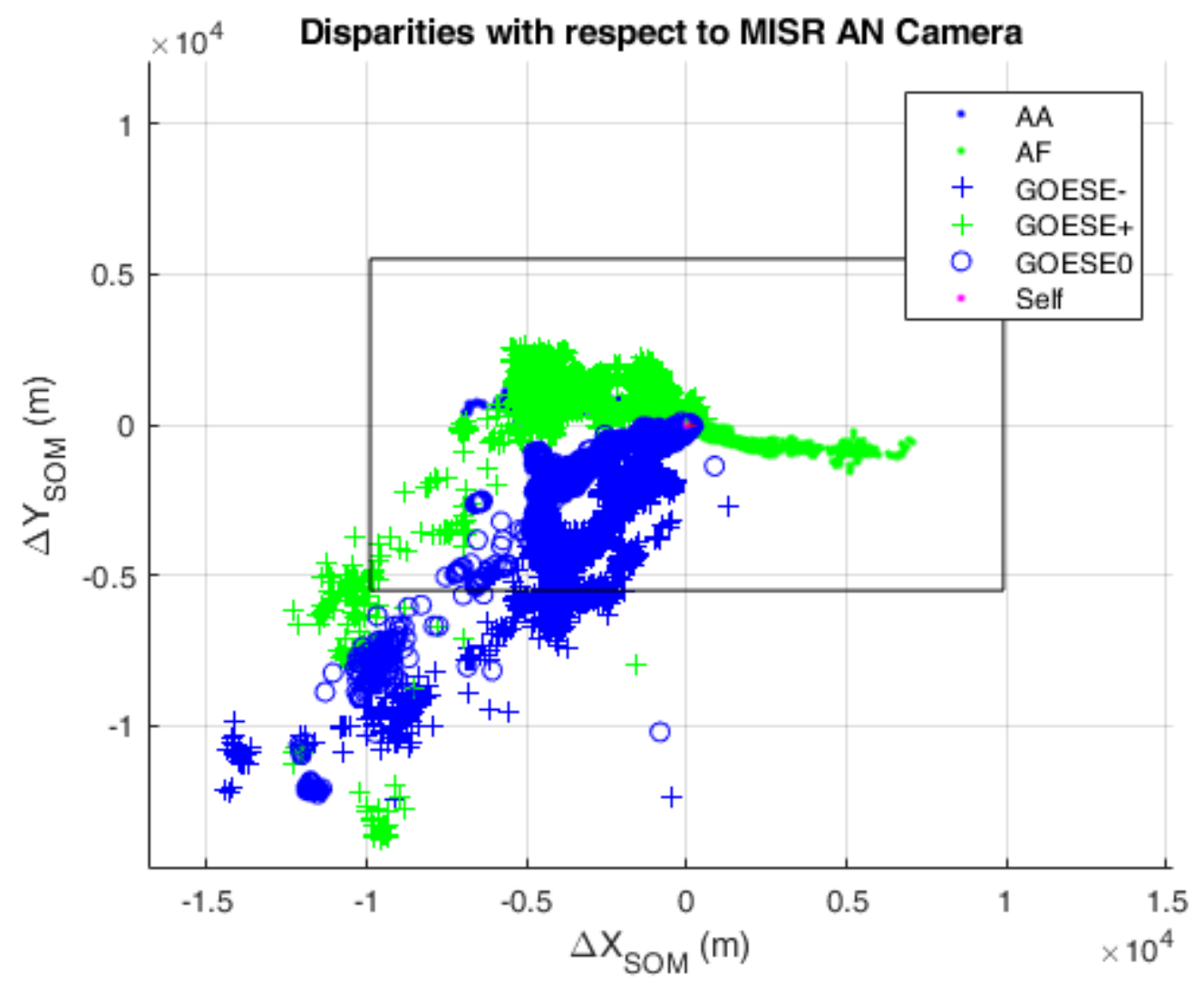

Figure 6. Prescreened disparities in the SOM projection for a single block. The GOES-to-An disparities are typically larger as they include both parallax and motion over a longer refresh period. Three repetitions of the GOES-16 scene are used ("GOESE0" most simultaneous, "GOESE-" trailing, "GOESE+" leading). The search space for MISR-MISR matches is outlined in black and has been sized to accommodate a parallax of up to $10 \mathrm{~km}$. The search space for GOES-MISR matches is larger to accommodate both parallax and winds up to $80 \mathrm{~m} / \mathrm{s}$.

Figure 7 provides the statistics for the residual disparities after being fit by the retrieval model. Note that the disparities shown in Figure 6 of up to $10 \mathrm{~km}$ now are well within the resolution elements of MISR and ABI B02, $275 \mathrm{~m}$ and $500 \mathrm{~m}$ respectively. Small systematic errors remain that cannot be modeled with the model states used and appear as biases in the residuals and not doubt also in the state solutions. 

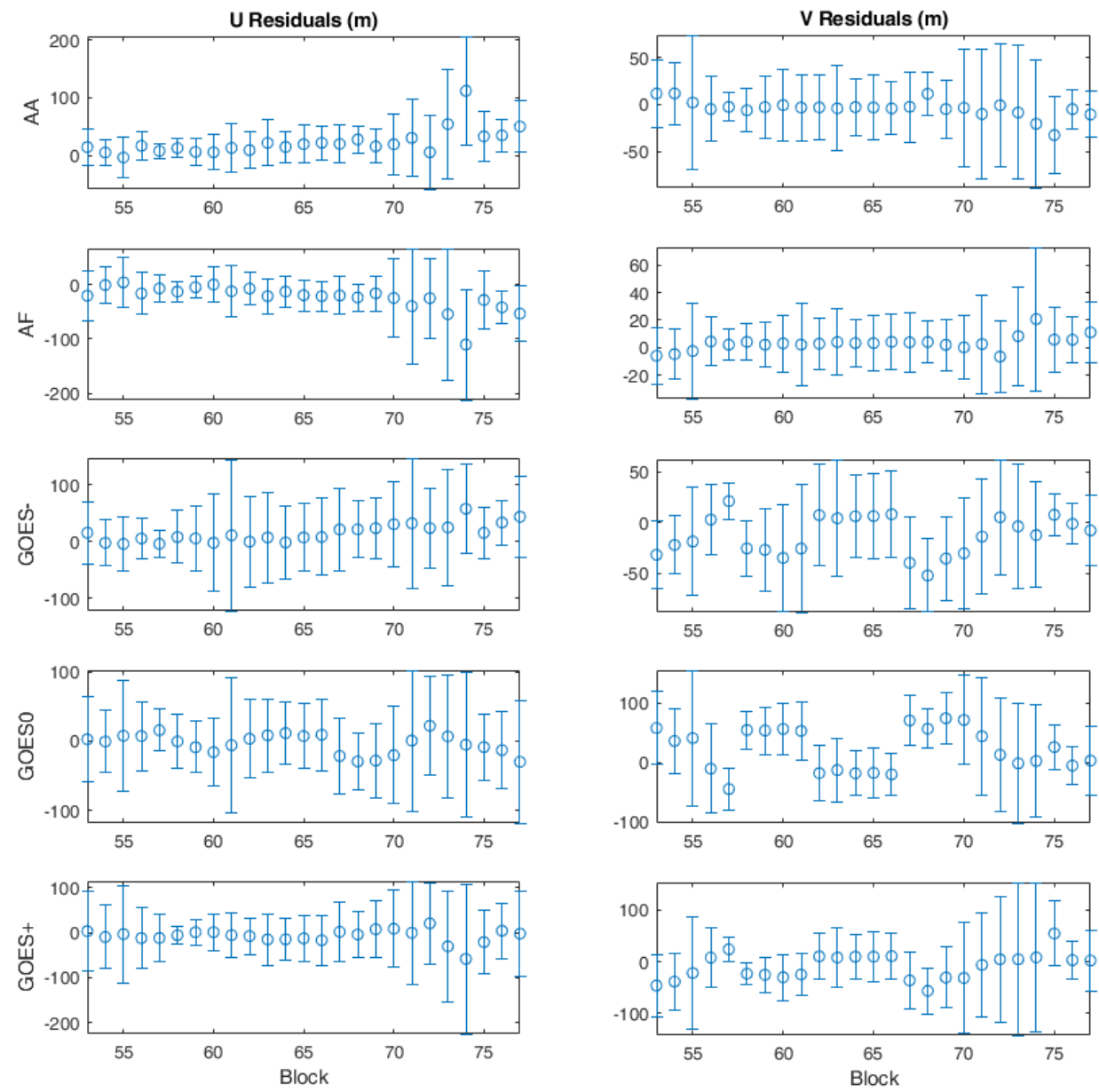

Figure 7. Residual disparities in the zonal (U) and meridional (V) wind coordinates for P024O098787B53:77 and GOES-16. The U coordinate is defined so that it increases to the east and the $\mathrm{V}$ coordinate is defined so that it increases to the north as measured in the tangent plane at each site. Each symbol designates the mean and one-sigma range. The mean value and standard deviation of residual systematic errors in each block are shown as a function of MISR block number.

The 3D-Wind algorithm has been evaluated with simulated disparities in a truth-in-truth-out test. We take a real AMV solution for one block and assume that as truth. We then calculate the disparities as they would be without errors and add known biases and normally distributed random errors to create a test dataset with known truth states and error statistics. If no errors are applied to the simulated disparities, the retrieval converges within a tolerance of $10 \mathrm{~cm}$ in position and $1 \mathrm{~cm} / \mathrm{s}$ in velocity in typically three iterations and the retrieved states are virtually indistinguishable from the simulated truth states. We can use the simulated datasets to demonstrate the importance of the bundle adjustment for compensating systematic errors that might affect retrievals and introduce systematic biases in the retrieved heights, and to estimate the accuracy of the AMV products given a statistical model for the uncertainties in the disparities. Figure 8 shows, as an example, histograms of simulated retrieval errors with and without the bundle adjustment in the presence of a systematic offset of the MISR imagery relative to that of GOES-16. The same offset has been applied to all A cameras, which are the only MISR cameras used. This situation would be similar to that of a CubeSat mission without the same level of fine pointing capability of an observatory-class mission such as Terra or GOES-R and where geo-registration errors might be significant but correlated between looks that are only tens of seconds apart. Such offsets induce systematic biases in the retrieved height but not the retrieved wind speeds. The bias is effectively compensated when the bundle adjustment is part of the solution and the bundle adjustment accurately estimates the offset that was applied. This is an important 
algorithm feature for future applications. Effectively, in such cases, the GEO system calibrates the INR of the LEO system and the fact that the LEO system's errors are correlated over a short period of time allows the LEO system to provide accurate information about cloud height in the presence of an offset. Small biases in the velocity retrievals can also be induced when there is a relative offset in the geo-registration of one of the members of the GEO triplet relative to the others.
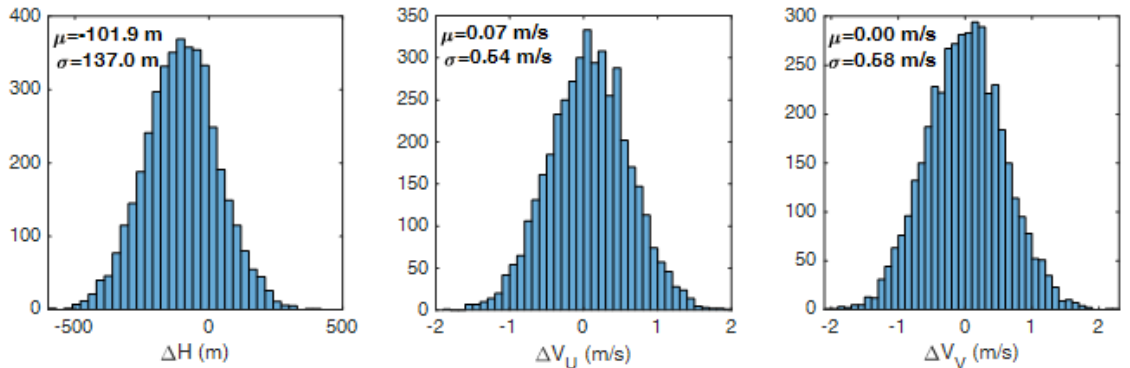

(a) No Bundle Adjustment
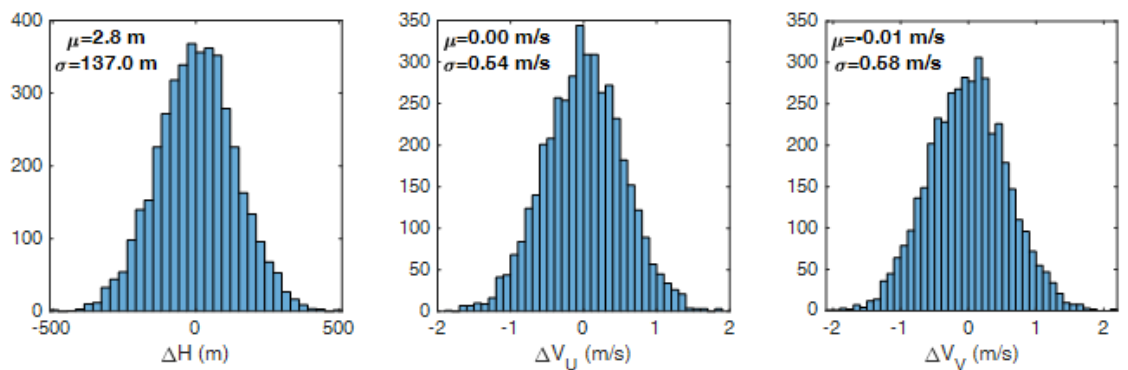

(b) Bundle Adjustment Applied

Figure 8. Distributions of retrieval errors (a) without and (b) with bundle adjustments from a Monte Carlo simulation. (U, V)-offsets were applied of $(100 \mathrm{~m},-150 \mathrm{~m})$ at each site in P024O098797B61, with a retrieved bundle-adjustment of (103.4 m, $-155.9 \mathrm{~m})$. The means $(\mu)$ and standard deviations $(\sigma)$ for each histogram are noted.

\subsubsection{Results and Validation}

The AMV and height retrievals from the 3D-Wind algorithm are validated and compared with MISR and GOES-16 operational Level-2 wind products in several different configurations. We first consider the MISR A cameras only and pair MISR data with GOES CONUS scenes. Later, we consider pairings with GOES MESO scenes and the impact of adding the MISR B, C and D cameras.

\section{Clear-Sky Retrievals over Terrain}

We validate retrievals over terrain under clear skies, where the retrieved velocities should be zero and height assignments match the ellipsoid height of the terrain that we derive from the GLOBE digital elevation model [50] with the EGM96 geoid [51]. The measurement of registration between clear-sky terrain imagery for validation purposes is a technique that has been pioneered with MISR [52]. To be classified as a clear-sky terrain retrieval, the retrieval site must be over land, apparently close to the ground $(<300 \mathrm{~m})$ and stationary (within $0.3 \mathrm{~m} / \mathrm{s}$ of zero in both $\mathrm{U}$ and $\mathrm{V}$ axes). Table 1 summarizes the clear-sky statistics for retrievals over P024 and P040 using either GOES-16 or -17. P008 is not considered as it is mostly over water while passing over the GOES CONUS scene and therefore its clear-sky terrain statistics are sparse. All paths traverse land with terrain heights ranging from zero to $\sim 3 \mathrm{~km}$, therefore, sampling retrieved heights up to $\sim 3 \mathrm{~km}$ but necessarily only stationary targets. The clear-sky terrain data for P024O098797 are shown in Figure 9. This path traverses the Great Plains, Gulf of Mexico, and a small mountainous portion of Central America as can be seen in Figure 4a. All retrievals below $\sim 5 \mathrm{~km}$ and not over water have been plotted versus the underlying terrain height. 
The separation of the clear-sky class from the other data is rather obvious in Figure $9 \mathrm{~b}$ and the clear-sky class falls cleanly on a regression line with slope $\sim 1$. Errors in the height retrievals are estimated from this class by simply differencing the retrieved height from the terrain height. The difference histogram resembles a normal distribution (Figure 9a). For the velocity error statistics (Figure 9c,d), the class height limit is adjusted to be $3 \sigma$ above the mean height error (apparent true ground level) and apparent velocity bounds are relaxed from $0.3 \mathrm{~m} / \mathrm{s}$ to $2 \mathrm{~m} / \mathrm{s}$ to admit to the sample retrievals with larger errors in the relevant variable. There are generally small but statistically significant biases in the heights and velocities retrieved for these apparently stationary ground sites from which we can estimate that the pattern height and velocity errors for A-camera plus GOES-16/17 retrievals are respectively less than about $200 \mathrm{~m}$ and $0.5 \mathrm{~m} / \mathrm{s}$. Retrievals using cloud patterns instead of terrain may possess additional uncertainties, which is a point made by Lonitz and Horváth [53] in their analysis of MISR winds. P024 has better height retrieval statistics than P040. The latter is exclusively over mountains, and it is quite possible that the height-retrieval accuracy assessment has some dependence on the background type. Mountainous backgrounds may affect both the retrievals and their accuracy assessment as one finds variations in elevation within templates and orographic clouds.

Table 1. Error statistics of clear-sky terrain height retrieval from the 3D-Wind algorithm. (Note that GOES-17 is providing preliminary, non-operational data while in the test slot at $89.5^{\circ} \mathrm{W}$.).

\begin{tabular}{cccccccccc}
\hline & & \multicolumn{3}{c}{ Regression } & \multicolumn{2}{c}{ Terrain } & \multicolumn{2}{c}{ Errors } \\
\hline $\begin{array}{c}\text { MISR } \\
\text { Path+Orbit }\end{array}$ & GOES & $\mathbf{N}_{\text {terrain }}$ & Slope & $\begin{array}{c}\text { Offset } \\
(\mathbf{m})\end{array}$ & $\mathbf{R}^{\mathbf{2}}$ & $\mathbf{1 \%}(\mathbf{m})$ & $\begin{array}{c}\mathbf{9 9 \%} \\
(\mathbf{m})\end{array}$ & $\begin{array}{c}\boldsymbol{\mu}(\boldsymbol{\Delta H}) \\
(\mathbf{m})\end{array}$ & $\begin{array}{c}\boldsymbol{\sigma}(\boldsymbol{\Delta H}) \\
(\mathbf{m})\end{array}$ \\
\hline P008O098097 & 16 & water & - & - & water & - & - & - & - \\
P024O098098 & 16 & 30,983 & 0.9984 & 11.7 & 0.9864 & -2.7 & 2676.8 & 11.1 & 68.3 \\
P040O098099 & 16 & 26,716 & 0.9817 & 51.0 & 0.9467 & -81.3 & 2367.9 & 35.7 & 126.9 \\
P008O098796 & 16 & water & - & - & water & - & - & - & - \\
P008O098796 & 17 & water & - & - & water & - & - & - & - \\
P024O098797 & 16 & 9606 & 0.9904 & -0.7 & 0.9893 & 3.8 & 2894.4 & -8.0 & 95.2 \\
P024O098797 & 17 & 4695 & 1.0725 & 2.2 & 0.9031 & -0.2 & 409.5 & 9.2 & 45.5 \\
P040O098798 & 16 & 30,138 & 0.9273 & 165.4 & 0.9230 & 278.1 & 2566.7 & 60.1 & 128.6 \\
P040O098798 & 17 & 55,557 & 0.9710 & 76.8 & 0.9513 & 16.5 & 2678.5 & 34.9 & 123.6 \\
\hline
\end{tabular}

(a) Retrieved ellipsoid heights compared to clear-sky over terrain heights. In each case, parameters are given for the regression of retrieved height versus terrain height as well as the sample size $\left(\mathrm{N}_{\text {terrain }}\right)$ and $1 \%$-to- $99 \%$ range of terrain heights. Sample means $(\mu)$ and standard deviations $(\sigma)$ of the differences between the retrieval and the terrain heights $(\Delta \mathrm{H})$ indicate the accuracy of the height retrievals.

\begin{tabular}{ccccccc}
\hline $\begin{array}{c}\text { MISR } \\
\text { Path+Orbit }\end{array}$ & GOES & $\mathbf{N}_{\text {terrain }}$ & $\mu\left(\mathbf{V}_{\mathbf{U}}\right)(\mathbf{m} / \mathbf{s})$ & $\sigma\left(\mathbf{V}_{\mathbf{U}}\right)(\mathbf{m} / \mathbf{s})$ & $\mu\left(\mathbf{V}_{\mathbf{V}}\right)(\mathbf{m} / \mathbf{s})$ & $\sigma\left(\mathbf{V}_{\mathbf{V}}\right)(\mathbf{m} / \mathbf{s})$ \\
\hline P008O098097 & 16 & water & - & - & - & - \\
P024O098098 & 16 & 33,414 & -0.03 & 0.12 & -0.00 & 0.18 \\
P040O098099 & 16 & 29,256 & -0.08 & 0.14 & 0.04 & 0.15 \\
P008O098796 & 16 & water & - & - & - & - \\
P008O098796 & 17 & water & - & - & - & - \\
P024O098797 & 16 & 10,044 & -0.01 & 0.11 & 0.09 & 0.12 \\
P024O098797 & 17 & 9412 & -0.26 & 0.40 & -0.04 & 0.13 \\
P040O098798 & 16 & 61,125 & -0.06 & 0.13 & -0.19 & 0.27 \\
P040O098798 & 17 & 61,770 & -0.14 & 0.10 & 0.12 & 0.10 \\
\hline
\end{tabular}

(b) Retrieved AMVs for clear-sky over terrain. In each case, the sample mean $(\mu)$ and standard deviation $(\sigma)$ statistics for the AMV components of clear-sky terrain retrievals are presented. Since these are presumably stationary objects, such statistics are indicative of the accuracy of the retrieved velocities. 

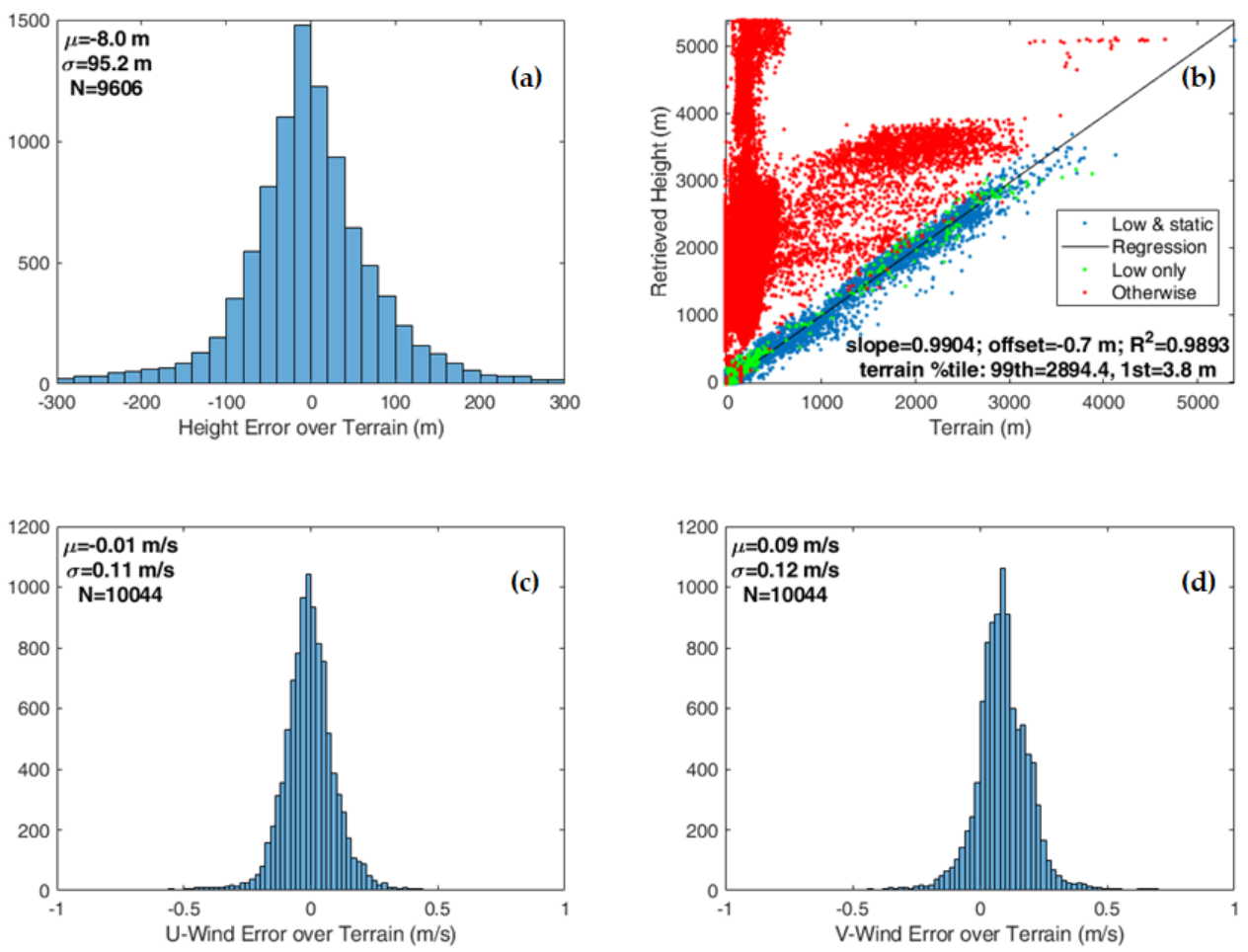

Figure 9. Error statistics for clear-sky retrievals over Terrain (P024O098797). Panels (a,c,d) show histograms for the height and velocity error samples. Panel (b) plots retrieved height versus terrain height. Retrievals over clear-sky terrain should fall along its diagonal. Low and static sites as identified in the legend comprise the class for validating height assignments, which is broadened to include additional low sites for velocity validation.

Comparisons with GOES Level-2 Derived Motion Winds

A comparison of the AMVs retrieved by the 3D-Wind algorithm against the GOES Level-2 Derived Motion Wind (DMW) product is shown in Figure 10 and Table 2 summarizes the difference statistics for the nine MISR paths. Each DMW is paired with its nearest 3D-Wind retrieval but accepting only pairings that are within $2.2 \mathrm{~km}$ of each other. In general, there are many more 3D-Wind AMVs than DMW vectors over the same area, which means that the number of pairings will be much smaller than the total number of 3D-Wind retrievals (Table 2). This comparison is made with the B02 DMWs but GOES also offers DMW products in three WV and two IR bands. It is not surprising that the paired GOES DMW velocities agree well with the 3D-Wind AMVs since both should be tracking the same patterns in the same spectral band. The more interesting comparison is in height assignments, which are given as pressures in the GOES DMW product. We converted the 3D-Wind heights into pressures and conversely the DMW pressures into heights using pressure profiles from the Modern-Era Retrospective analysis for Research and Applications, Version 2 (MERRA-2) reanalysis system [54]. Figure 11 shows the pressure and height assignment comparisons. We added Band 14 (B14) DMWs so more upper-level winds could be included, but it is likely that in some cases the B02 and B14 DMWs are tracking different features. Many AMVs are assigned to low altitudes by the 3D-Winds algorithm but are paired with winds near the tropopause in the GOES B14 DMW product possibly because cirrus clouds that are semitransparent in B02 are being tracked in B14. This highlights the potential that IR temperatures from one layer could be used to assign heights to AMVs in another.

The statistics comparing the height assignments from the B02 DMWs and the 3D-Wind algorithm are found in Table 3, which indicates that height assignment differences are $\sim 1 \mathrm{~km}$ and biased low. Most of the DMWs compared are low in the atmosphere (below $\sim 3 \mathrm{~km}$ ). Given that $\sim 100 \mathrm{~m}$ error is typical over clear-sky terrain for the MISR+GOES 3D-Wind algorithm, it is expected that the 
3D-Wind algorithm's height assignments are significantly more accurate than those of the operational GOES DMW product, especially at low altitudes (consistent with findings with for Meteosat-9 and boundary-layer clouds [53]). To assess the resulting improvement in forecast skill from the more accurate height assignments would require a large ensemble to conduct an impact study as in $[30,55]$.
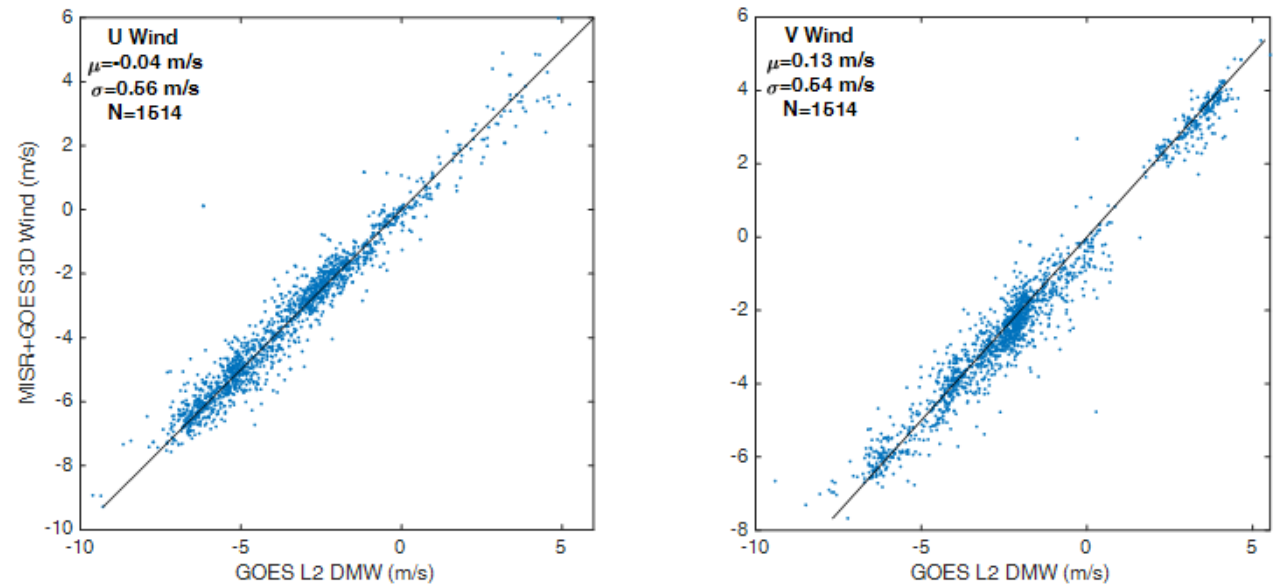

Figure 10. Comparison of AMVs for the MISR+GOES 3D-Winds algorithm for P024O098098 and GOES-16 L2 DMWs. There are 122,339 AMVs along the path of which 1514 have been paired with CONUS visible (B02) DMWs. Diagonal lines are lines of equal wind velocity.

Table 2. Comparison of the 3D-Winds retrievals and GOES L2 DMWs. Each case is summarized by the total number of 3D-Wind retrievals over CONUS $\left(\mathrm{N}_{3 \mathrm{D}}\right)$, the number of them paired with a DMW $\left(\mathrm{N}_{\text {Paired }}\right)$, and the means $(\mu)$ and standard deviations $(\sigma)$ of the velocity differences. (Note that GOES-17 is providing preliminary, non-operational data while in the test slot at $89.5^{\circ} \mathrm{W}$.).

\begin{tabular}{cccccccc}
\hline $\begin{array}{c}\text { MISR } \\
\text { Path+Orbit }\end{array}$ & GOES & $\mathbf{N}_{3 D}$ & $\mathbf{N}_{\text {Paired }}$ & $\begin{array}{c}\mu\left(\Delta \mathbf{V}_{\mathbf{U}}\right) \\
(\mathbf{m} / \mathbf{s})\end{array}$ & $\begin{array}{c}\sigma\left(\Delta \mathbf{V}_{\mathbf{U}}\right) \\
(\mathbf{m} / \mathbf{s})\end{array}$ & $\begin{array}{c}\mu\left(\Delta \mathbf{V}_{\mathbf{V}}\right) \\
(\mathbf{m} / \mathbf{s})\end{array}$ & $\begin{array}{c}\sigma\left(\Delta \mathbf{V}_{\mathbf{V}}\right) \\
(\mathbf{m} / \mathbf{s})\end{array}$ \\
\hline P008O098097 & 16 & 85,461 & 715 & 0.22 & 0.81 & 0.30 & 0.87 \\
P024O098098 & 16 & 122,339 & 1514 & -0.04 & 0.56 & 0.13 & 0.54 \\
P040O098099 & 16 & 59,731 & 90 & -0.02 & 1.04 & 0.47 & 1.06 \\
P008O098796 & 16 & 76,791 & 2391 & -0.04 & 0.55 & 0.07 & 0.54 \\
P008O098796 & 17 & 76,869 & 2397 & -0.17 & 0.59 & 0.22 & 0.57 \\
P024O098797 & 16 & 111,894 & 941 & 0.01 & 0.48 & 0.23 & 0.56 \\
P024O098797 & 17 & 112,501 & 941 & -0.17 & 0.48 & 0.22 & 0.59 \\
P040O098798 & 16 & 71,409 & 31 & 0.27 & 1.67 & -0.32 & 0.88 \\
P040O098798 & 17 & 72,025 & 30 & 0.12 & 1.72 & -0.13 & 0.75 \\
\hline
\end{tabular}

Table 3. Statistics of the 3D-Wind and GOES DMW height/pressure differences. Each case is summarized by the number of parings between 3D-Wind retrievals and DMW vectors $\left(\mathrm{N}_{\text {Paired }}\right)$, and the means $(\mu)$ and standard deviations $(\sigma)$ of their pressure differences $(\Delta \mathrm{P})$ and height differences $(\Delta \mathrm{H})$. (Note that GOES-17 is providing preliminary, non-operational data while in the test slot at $89.5^{\circ} \mathrm{W}$.).

\begin{tabular}{ccccccc}
\hline $\begin{array}{c}\text { MISR } \\
\text { Path+Orbit }\end{array}$ & GOES & $\mathbf{N}_{\text {Paired }}$ & $\begin{array}{c}\mu(\Delta \mathbf{P}) \\
(\mathbf{h P a})\end{array}$ & $\begin{array}{c}\sigma(\Delta \mathbf{P}) \\
(\mathbf{h P a})\end{array}$ & $\begin{array}{c}\mu(\boldsymbol{\Delta H}) \\
(\mathbf{m})\end{array}$ & $\begin{array}{c}\sigma(\Delta \mathbf{H}) \\
(\mathbf{m})\end{array}$ \\
\hline P008O098097 & 16 & 715 & 43.1 & 70.7 & -419.8 & 732.1 \\
P024O098098 & 16 & 1514 & 93.5 & 38.6 & -936.8 & 392.2 \\
P040O098099 & 16 & 90 & 116.0 & 75.8 & -1332.3 & 863.4 \\
P008O098796 & 16 & 2391 & 65.6 & 60.3 & -628.8 & 600.3 \\
P008O098796 & 17 & 2397 & 67.1 & 59.9 & -644.1 & 597.3 \\
P024O098797 & 16 & 941 & 34.8 & 60.2 & -356.1 & 611.2 \\
P024O098797 & 17 & 941 & 34.2 & 60.0 & -349.5 & 608.5 \\
P040O098798 & 16 & 31 & 16.9 & 28.3 & -145.4 & 351.8 \\
P040O098798 & 17 & 30 & 19.8 & 27.7 & -172.9 & 345.2 \\
\hline
\end{tabular}



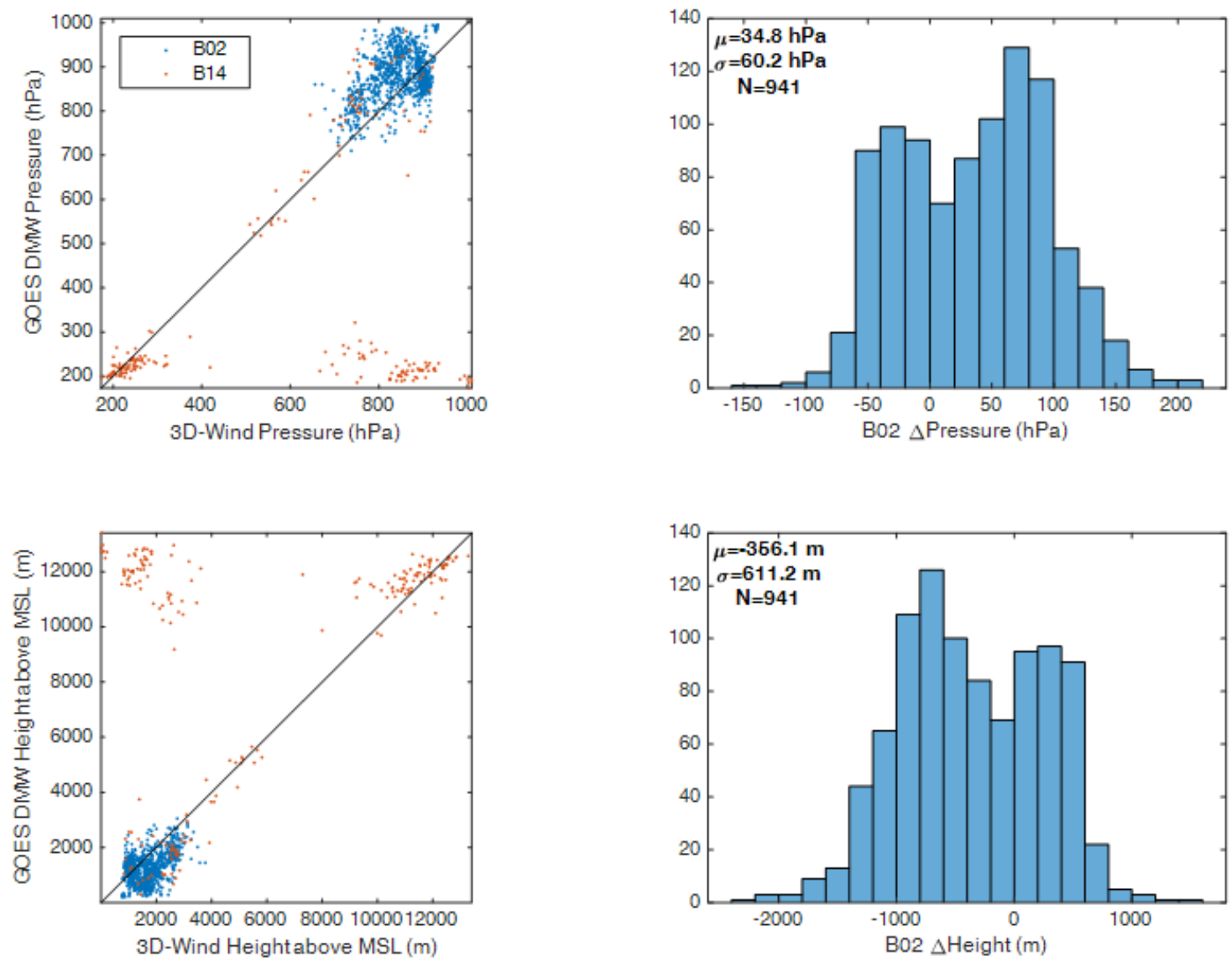

Figure 11. Comparison of the 3D-Wind and GOES DMW height/pressure assignments The MERRA-2 M2T3NVASM (time-averaged assimilated meteorological fields) collection includes pressure versus height above Mean Sea Level (MSL) at 72 model layers on a $0.5^{\circ} \times 0.625^{\circ}$ latitude-longitude grid every $3 \mathrm{~h}$ that permits conversion between pressure and height above MSL. Diagonal lines are lines of equal pressure or height.

Comparisons with MISR Winds

The 3D-Wind algorithm is expected to improve on MISR wind products as well because of the additional constraints placed on the AMVs by the GOES observations. We compare the operational MISR wind product (TC_CLOUD) with the MISR+GOES 3D-Wind AMVs in an example as shown in Figure 12. The statistics of the differences are summarized in Table 4. The two products are horizontally sampled on different grids, $17.6 \mathrm{~km}$ for the former and $2.2 \mathrm{~km}$ for the latter. To make the comparison, all 3D-Wind AMVs within $5 \mathrm{~km}$ of each $17.6 \mathrm{~km}$ sample site are averaged. The $\mathrm{U}$ winds match reasonably well, but not so for the $\mathrm{V}$ winds. For MISR, the $\mathrm{V}$ wind is mostly along track and the $\mathrm{U}$ wind is mostly cross track. It is not surprising that MISR-only height and V winds are scattered more when compared to the MISR+GOES 3D-Wind retrievals winds since coupling between height and along-track wind is a known challenge for MISR alone. Most V-wind differences have small means and large variances, but $\mathrm{P} 008 \mathrm{O} 098097$ is an outlier with a larger bias for unknown reasons. There is a clear indication from the maps in Figure 12 that the V-wind difference and height difference are highly correlated. Although the height assignments appear to match better than to those of the GOES DMWs, the differences are still significantly larger than would be expected from the 3D-Wind error statistics derived over clear-sky terrain (Table 1) that indicates a height assignment accuracy in the range of 100-200 m. 

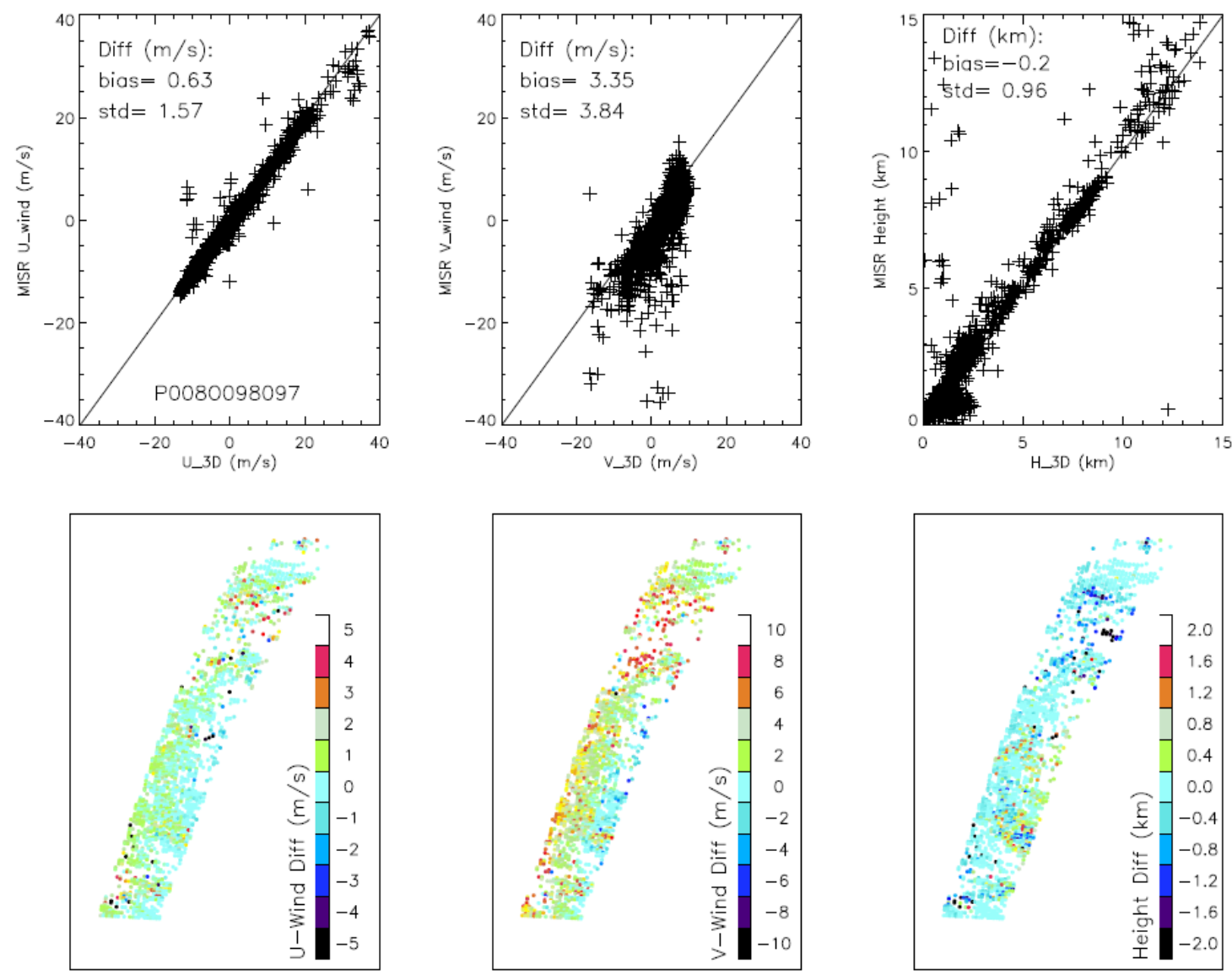

Figure 12. Top: Comparison of the $\mathrm{U}, \mathrm{V}$, and $\mathrm{H}$ retrievals between the MISR+GOES 3D-Wind and the MISR-only algorithms from a single path (P008O098097); Bottom: Differences (3D-Wind minus MISR-only) of the U, V, H retrievals between two algorithms.

Table 4. Comparison Statistics between the 3D-Wind and the MISR-only AMV Products. Each case is summarized by the means $(\mu)$ and standard deviations $(\sigma)$ of the height differences $(\Delta H)$ and velocity differences $(\Delta \mathrm{V})$. (Note that GOES-17 is providing preliminary, non-operational data while in the test slot at $89.5^{\circ} \mathrm{W}$.).

\begin{tabular}{|c|c|c|c|c|c|c|c|}
\hline $\begin{array}{c}\text { MISR } \\
\text { Path+Orbit }\end{array}$ & GOES & $\begin{array}{c}\mu(\Delta \mathrm{H}) \\
(\mathrm{km})\end{array}$ & $\begin{array}{c}\sigma(\Delta \mathrm{H}) \\
(\mathbf{k m})\end{array}$ & $\begin{array}{c}\mu\left(\Delta \mathrm{V}_{\mathrm{U}}\right) \\
(\mathrm{m} / \mathrm{s})\end{array}$ & $\begin{array}{c}\sigma\left(\Delta \mathrm{V}_{\mathrm{U}}\right) \\
(\mathrm{m} / \mathrm{s})\end{array}$ & $\begin{array}{c}\mu\left(\Delta V_{V}\right) \\
(\mathrm{m} / \mathrm{s})\end{array}$ & $\begin{array}{c}\sigma\left(\Delta \mathrm{V}_{\mathrm{V}}\right) \\
(\mathrm{m} / \mathrm{s})\end{array}$ \\
\hline P0080098097 & 16 & -0.2 & 0.96 & 0.63 & 1.57 & 3.35 & 3.84 \\
\hline P024O098098 & 16 & 0.2 & 0.63 & -0.21 & 1.30 & -0.52 & 2.52 \\
\hline P040O098099 & 16 & 0.1 & 0.72 & 0.30 & 1.31 & 0.33 & 3.98 \\
\hline P008O098796 & 16 & 0.0 & 0.77 & 0.26 & 1.35 & 0.94 & 3.30 \\
\hline P008O098796 & 17 & 0.0 & 0.77 & 0.34 & 1.33 & 0.75 & 3.30 \\
\hline P024O098797 & 16 & 0.1 & 0.58 & 0.08 & 1.15 & 0.14 & 3.43 \\
\hline P024O098797 & 17 & 0.1 & 0.63 & 0.15 & 1.15 & 0.10 & 3.40 \\
\hline P040O098798 & 16 & 0.2 & 0.74 & 0.07 & 0.88 & 0.27 & 1.62 \\
\hline P040O098798 & 17 & 0.1 & 0.70 & -0.01 & 0.70 & 0.57 & 1.61 \\
\hline
\end{tabular}

\section{MISR with GOES MESO Scenes}

MISR paths can also be paired with GOES full-disk and MESO scenes. The refresh period for MESO scenes can be either one minute or $30 \mathrm{~s}$ and generally $15 \mathrm{~min}$ for a full disk. The rapid refresh for a MESO scene is an interesting capability for following highly dynamic meteorological phenomena such as hurricanes. With the more rapid refresh, there is greater assurance that one of the fundamental assumptions of AMVs, that a tracked feature remains invariant and only translates, is valid. This is of 
course traded off against the shorter time between refresh cycles, so whatever uncertainty exists in subpixel measurements of feature displacement will have greater effect on the uncertainty in retrieved wind velocity. We have yet to fully assess the implications of this tradeoff on the 3D-Winds algorithm, but we have experimented with 3D-Wind retrievals using MISR and GOES MESO 30-s imagery. Figure 13a is an example from Hurricane Florence with a very high density of successful retrievals. We can clearly see the low-altitude cyclonic winds to the south of the eye that feed the storm with warm moist air from the boundary layer and high-altitude anticyclonic winds to the north carrying away cooler air.

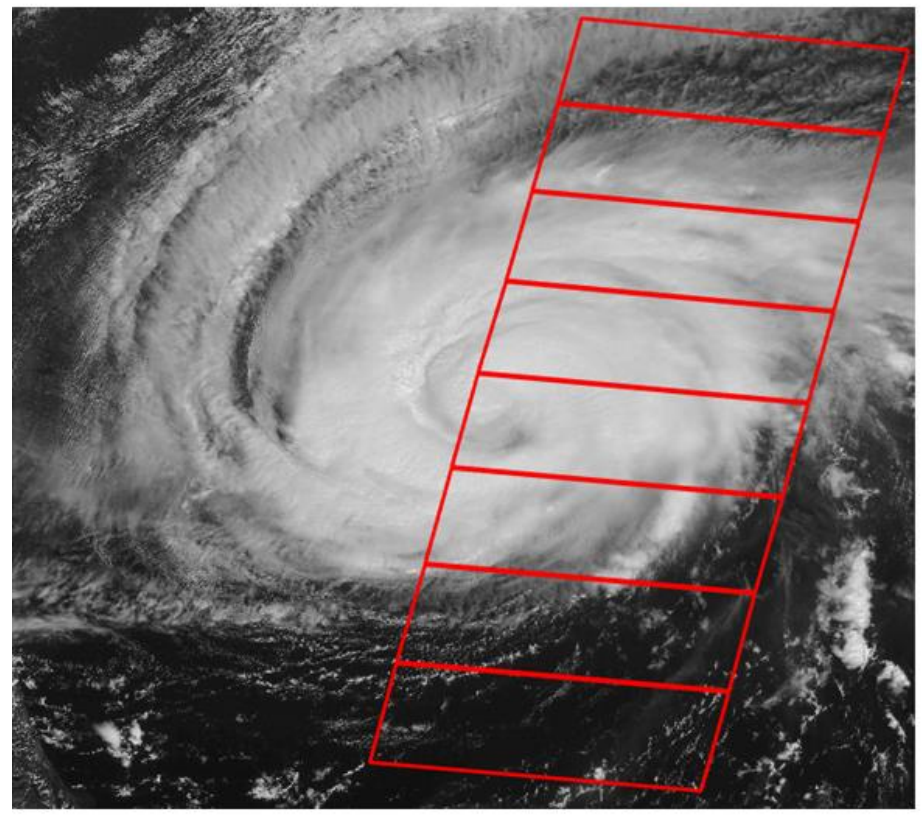

(a) GOES-16B02

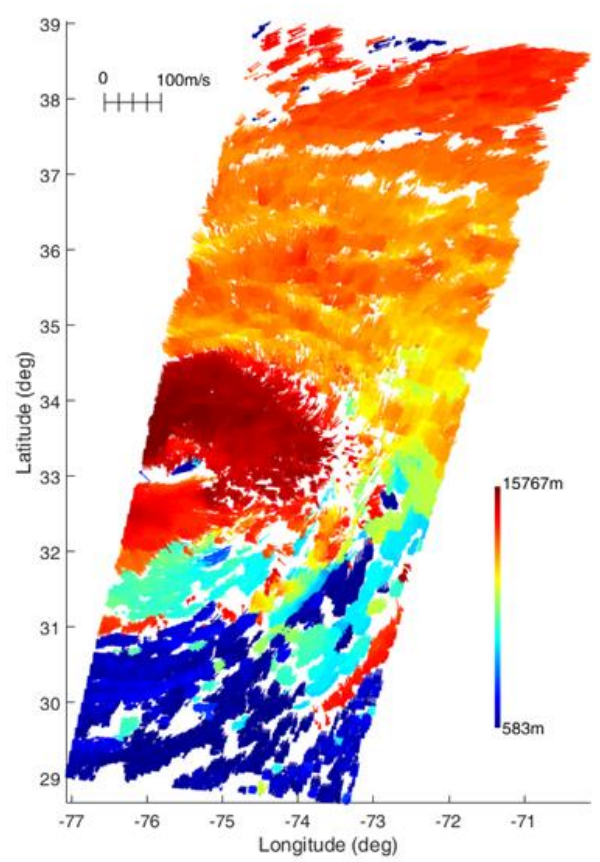

(b) $3 \mathrm{D}$ Winds

Figure 13. MESO image of (a) Hurricane Florence with (b) retrieved 3D-Winds.

Comparing the 3D-Wind algorithm retrievals with MISR winds in the Florence case shows the same pattern as before with a good match between $\mathrm{U}$ winds but a larger scatter in the V-wind comparison (Figure 14). The MISR level-2 algorithm has difficulty producing 17.6-km sampled AMVs over much of Hurricane Florence. The MISR algorithm requires the AMV and CTH quality to pass consistency checks between the independent retrievals from the fore and aft cameras [30]. Because the MISR-only retrieval employs the images from oblique angles, the consistency criteria may become difficult to pass in the presence of multi-layer clouds. The problem of multi-layer clouds, which may be in the northmost block, could cause large discrepancies between the 3D-Wind and MISR-only retrievals in AMV and CTH.

A strong correlation is found between the height difference and the $\mathrm{V}$-wind difference for all the MISR paths to which the 3D-Winds algorithm was applied. Figure 15 aggregates all collocated retrieval differences from the Hurricane Florence and the nine previous CONUS cases. Most of the height differences $<-2 \mathrm{~km}$ are from the Hurricane Florence path. The differences in AMV and height data fall along a line with a slope equal to $-100 \mathrm{~s}$, which is the approximate time between when the same feature is seen by the fore and aft A cameras. Theoretically, it is consistent with the expected relationship for the error correlation between MISR along-track wind and height [28,30]. A number of large positive $(>2 \mathrm{~km})$ height differences with a small V-wind difference come from near-surface clouds, which could be caused by different retrieval sampling distances $(17.6 \mathrm{~km}$ vs. $2.2 \mathrm{~km})$. Further investigation of the comparison between the MISR and 3D-Wind algorithm products would be desirable to explore this subject further with a large statistical ensemble. 

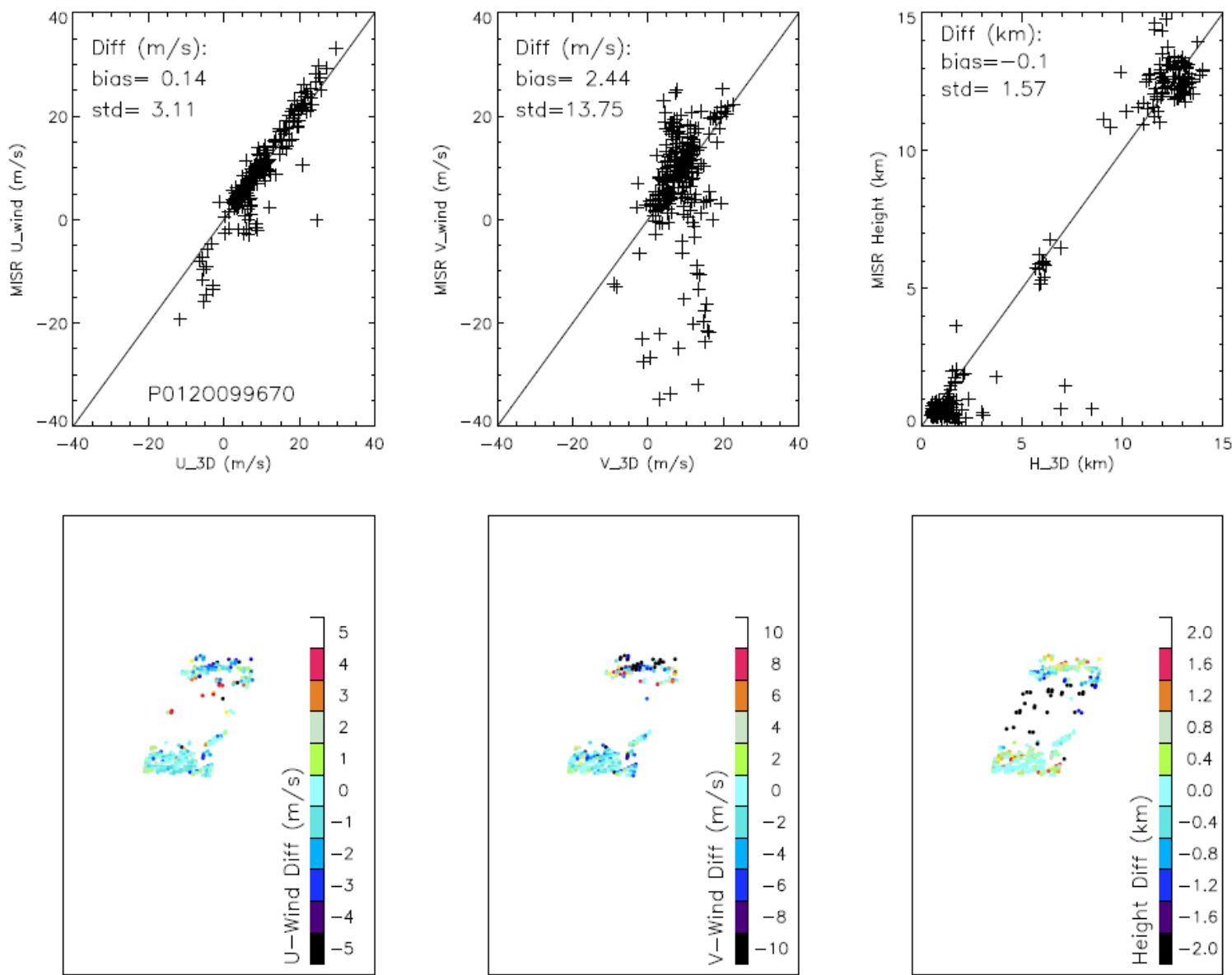

Figure 14. As in Figure 2 but for Hurricane Florence. The MISR standard level-2 products have very few retrievals above the hurricane deck, leaving an unexpectedly low number of comparisons for high-level clouds. Large negative V-wind differences, correlated with positive height differences, are evident in the northmost block where multi-layer clouds are present.

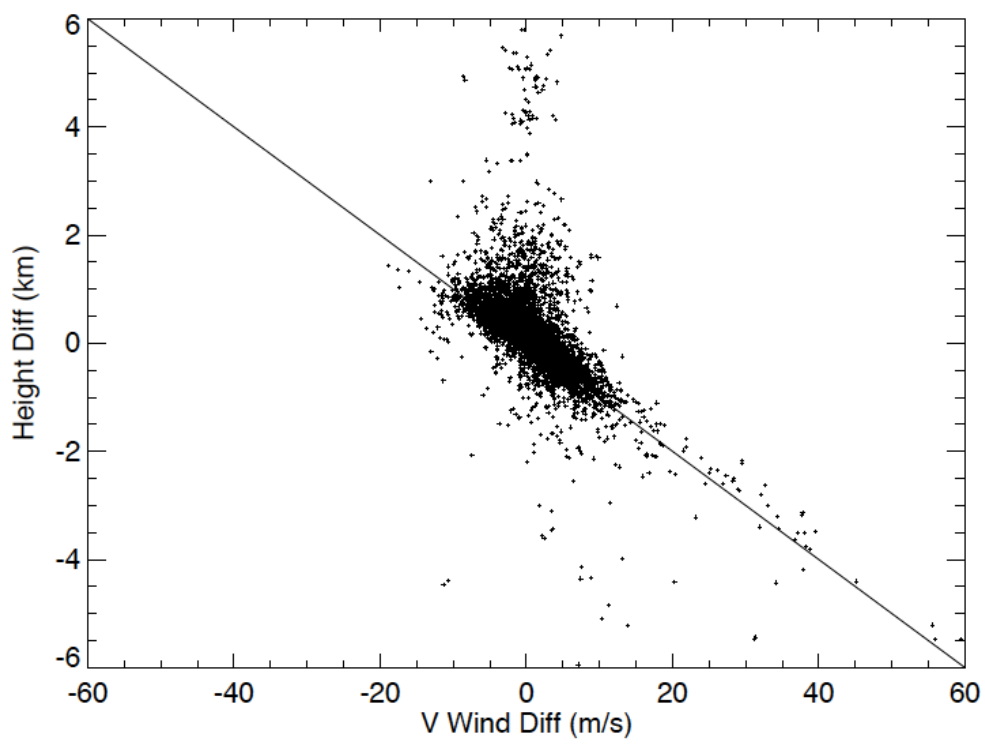

Figure 15. Correlated 3D-Wind and MISR-only differences in the height and V-wind retrievals for Hurricane Florence and all nine CONUS cases. 


\section{MISR B, C and D Cameras}

The 3D-Wind retrieval algorithm can also use images from the MISR B, C, and D cameras in addition to the A cameras. As more cameras are added, there are generally fewer successful retrievals. This is expected as there are more disparity pairings and thus more opportunities to fail one of the quality screening tests. Table 5 summarizes the statistics from two paths using progressively more MISR cameras. There appears to be some marginal benefit in height accuracy by adding the B cameras but thereafter the benefit of adding cameras diminishes and potentially the D cameras adversely affect the retrievals. The velocity statistics seems to be less sensitive to the addition of more cameras.

Table 5. Retrieval statistics over clear-sky terrain (MISR + GOES-16). Each case is summarized by the means $(\mu)$ and standard deviations $(\sigma)$ of the height differences $(\Delta \mathrm{H})$ and velocity differences $(\Delta \mathrm{V})$ for the number of clear-sky terrain retrievals $\left(\mathrm{N}_{\text {terrain }}\right)$ applicable to each out of the total population of all retrievals $\left(\mathrm{N}_{3 \mathrm{D}}\right)$.

\begin{tabular}{ccccccccccc}
\hline $\begin{array}{c}\text { MISR } \\
\text { Path+Orbit }\end{array}$ & Cameras & $\mathbf{N}_{3 \mathrm{D}}$ & $\begin{array}{c}\mathbf{N}_{\text {terrain }} \\
\text { for } \Delta \mathbf{H}\end{array}$ & $\begin{array}{c}\mu(\mathbf{\Delta H}) \\
(\mathbf{m})\end{array}$ & $\begin{array}{c}\sigma(\mathbf{\Delta H}) \\
(\mathbf{m})\end{array}$ & $\begin{array}{c}\mathbf{N}_{\text {terrain }} \\
\text { for } \mathbf{V}\end{array}$ & $\begin{array}{c}\mu\left(\mathbf{V}_{\mathrm{U}}\right) \\
(\mathbf{m} / \mathbf{s})\end{array}$ & $\begin{array}{c}\sigma\left(\mathbf{V}_{\mathbf{U}}\right) \\
(\mathbf{m} / \mathbf{s})\end{array}$ & $\begin{array}{c}\mu\left(\mathbf{V}_{\mathbf{V}}\right) \\
(\mathbf{m} / \mathbf{s})\end{array}$ & $\begin{array}{c}\sigma\left(\mathbf{V}_{\mathbf{V}}\right) \\
(\mathbf{m} / \mathbf{s})\end{array}$ \\
\hline P024O098797 & $\mathrm{A}$ & 111,894 & 9606 & -8.0 & 95.2 & 10,044 & -0.01 & 0.11 & 0.09 & 0.12 \\
P024O098797 & $\mathrm{AB}$ & 105,131 & 8952 & 13.4 & 82.9 & 9435 & 0.08 & 0.12 & 0.07 & 0.12 \\
P024O098797 & $\mathrm{ABC}$ & 90,230 & 7643 & 7.6 & 77.0 & 8152 & 0.08 & 0.12 & 0.07 & 0.12 \\
P024O098797 & $\mathrm{ABCD}$ & 69,249 & 5116 & 38.7 & 67.2 & 5310 & 0.06 & 0.12 & 0.01 & 0.09 \\
P040O098798 & $\mathrm{A}$ & 71,409 & 30,138 & 60.1 & 128.6 & 61,125 & -0.06 & 0.13 & -0.19 & 0.27 \\
P040O098798 & $\mathrm{AB}$ & 68,956 & 27,130 & 24.5 & 116.1 & 58,498 & -0.05 & 0.14 & -0.19 & 0.29 \\
P040O098798 & $\mathrm{ABC}$ & 61,826 & 25,324 & 22.9 & 106.8 & 53,395 & -0.07 & 0.15 & -0.19 & 0.29 \\
P040O098798 & $\mathrm{ABCD}$ & 50,403 & 18,720 & 40.6 & 95.4 & 43,730 & -0.00 & 0.17 & -0.22 & 0.29 \\
\hline
\end{tabular}

\section{W-Component Retrievals}

Here we consider relaxing the constraint on the AMV that it must lie in the horizontal plane and allow for a retrieval that includes a vertical or W-component (i.e., six states per site). It is a simple matter to demonstrate observability of all states from simulated data under similar assumptions used to make Figure 8. Whether the W-component is interpreted as an updraft/downdraft, growth/collapse of a cloud, or is an artifact of matching between imagery of vertically extended objects looking down on them and obliquely is a separate question that deserves careful attention in further research. Table 6 presents several simulation cases, each a variation on that of Figure 8. Case 1a is our baseline (exactly Figure 8) with two-component AMV retrievals and truth winds that are strictly horizontal. Case $1 \mathrm{~b}$ adds a $2 \mathrm{~m} / \mathrm{s} \mathrm{W}$-component to all truth winds to show that, if unmodeled, both the retrieved height and UV-components become biased. This bias is effectively removed when a $\mathrm{W}$-component is retrieved (Case 1c) but uncertainties grow in the other retrieved states, particularly in the AMVs. This shows from a strictly theoretical perspective that solving for three-component AMVs is not beneficial when the $\mathrm{W}$-components are truly nearly zero in nature, which is thought to be the majority of the time in nature. On the other hand, situations where the W-component is large ( $2 \mathrm{~m} / \mathrm{s}$ [56]) may be of meteorological significance and therefore should not be ignored. Adding additional MISR cameras adds to the observability of the W-component as shown in Cases 2, at least with the simplistic error model used in our simulation, and uncertainties in all AMV components approach baseline values when using the $A B C$ and $A B C D$ cameras. Adding a second GEO spacecraft (e.g., GOES-17) where there is an overlap to the joint retrieval should also influence observability, as well as the relative geometries of the participating LEO and GEO spacecraft. 
Table 6. Simulations of retrievals with six states at each site. Cases 1 use the A-cameras only with a W-component for all truth AMVs of either zero or $2 \mathrm{~m} / \mathrm{s}$. Cases 2 progressively add camera pairs. Each case is summarized by the means $(\mu)$ and standard deviations $(\sigma)$ of the height differences $(\Delta \mathrm{H})$ and velocity differences $(\Delta \mathrm{V})$ relative to simulation truth.

\begin{tabular}{ccccccccccc}
\hline Case & $\begin{array}{c}\text { MISR } \\
\text { Cameras }\end{array}$ & $\begin{array}{c}\mathbf{V}_{\mathbf{W}} \\
(\mathbf{m} / \mathbf{s})\end{array}$ & $\begin{array}{c}\mu(\mathbf{\Delta H}) \\
(\mathbf{m})\end{array}$ & $\begin{array}{c}\sigma(\mathbf{\sigma}) \\
(\mathbf{m})\end{array}$ & $\begin{array}{c}\mu\left(\mathbf{V}_{\mathbf{U}}\right) \\
(\mathbf{m} / \mathbf{s})\end{array}$ & $\begin{array}{c}\sigma\left(\mathbf{V}_{\mathbf{U}}\right) \\
(\mathbf{m} / \mathbf{s})\end{array}$ & $\begin{array}{c}\mu\left(\mathbf{V}_{\mathbf{V}}\right) \\
(\mathbf{m} / \mathbf{s})\end{array}$ & $\begin{array}{c}\sigma\left(\mathbf{V}_{\mathbf{V}}\right) \\
(\mathbf{m} / \mathbf{s})\end{array}$ & $\begin{array}{c}\mu\left(\mathbf{V}_{\mathbf{W}}\right) \\
(\mathbf{m} / \mathbf{s})\end{array}$ & $\begin{array}{c}\sigma\left(\mathbf{V}_{\mathbf{W}}\right) \\
(\mathbf{m} / \mathbf{s})\end{array}$ \\
\hline $1 \mathrm{a}$ & $\mathrm{A}$ & 0 & 2.8 & 137.0 & 0.00 & 0.54 & -0.01 & 0.58 & - & - \\
$1 \mathrm{~b}$ & $\mathrm{~A}$ & 2 & -144.5 & 137.3 & -0.85 & 0.54 & 1.84 & 0.58 & - & - \\
$1 \mathrm{c}$ & $\mathrm{A}$ & 2 & 4.4 & 174.0 & 0.01 & 1.36 & -0.03 & 2.56 & 0.02 & 2.71 \\
$2 \mathrm{a}$ & $\mathrm{A}$ & 0 & 4.4 & 174.0 & 0.01 & 1.36 & -0.03 & 2.56 & 0.02 & 2.71 \\
$2 \mathrm{~b}$ & $\mathrm{AB}$ & 0 & -3.3 & 113.8 & 0.00 & 0.66 & 0.01 & 1.19 & -0.01 & 1.21 \\
$2 \mathrm{c}$ & $\mathrm{ABC}$ & 0 & -1.3 & 73.5 & 0.00 & 0.42 & 0.02 & 0.70 & -0.01 & 0.50 \\
$2 \mathrm{~d}$ & $\mathrm{ABCD}$ & 0 & 0.7 & 52.4 & -0.01 & 0.32 & -0.01 & 0.57 & -0.01 & 0.22 \\
\hline
\end{tabular}

Guided by the simulation results, we use all nine MISR cameras and retrieve six states per site for P024O098797 and GOES-16 from real instead of simulated data. Figure 16 shows a map of retrieved $\mathrm{W}$-components, most of which are quite small in magnitude as would be expected and larger magnitudes seem to be associated with broken cloud fields. Figure 17 shows histograms for the differences between these retrievals and those of Figure $4 \mathrm{~b}$ (baseline case: A cameras and strictly horizontal AMVs) at sites where both had successful retrievals. First and second order statistics for the differences are within expectations given the estimated accuracy for the baseline case.

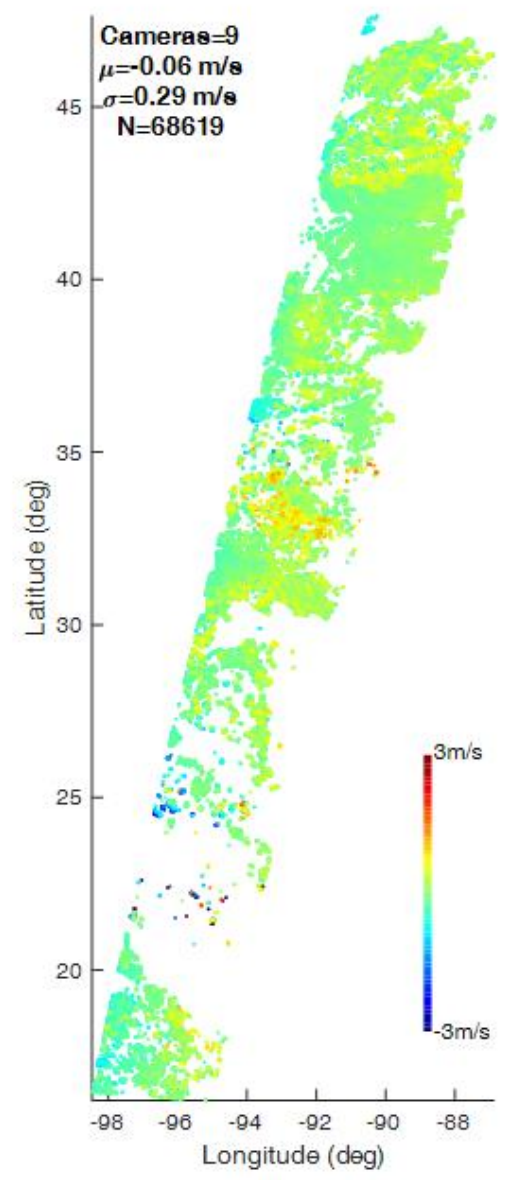

Figure 16. Retrieved W-components for P024O098797 using MISR ABCD Cameras. Retrieval sites are color coded to show the retrieved W-component. 

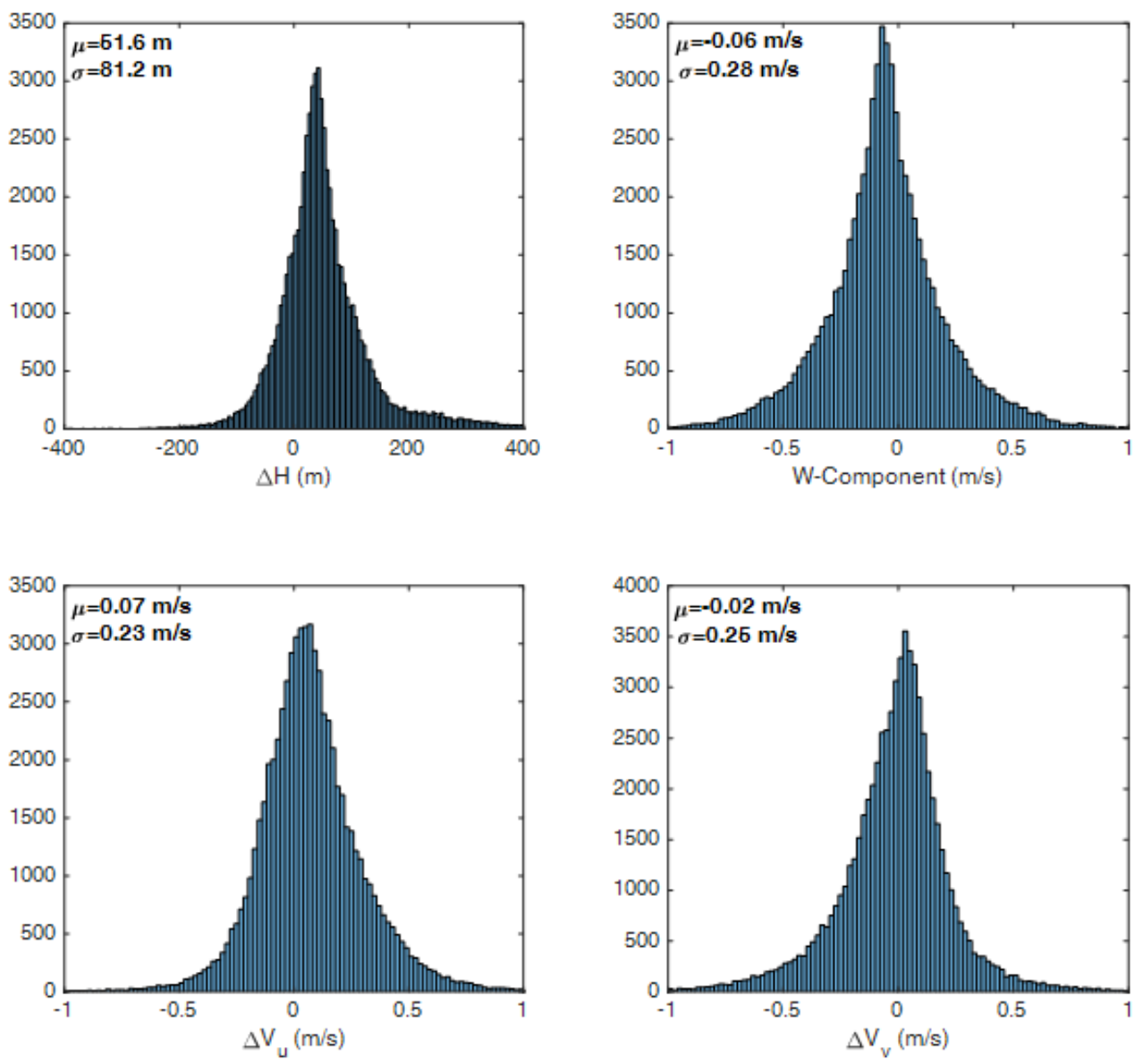

Figure 17. Statistics of the comparison between retrievals with six versus five states per site.

Summary of Validations and Comparisons

In summary, the validations above show that the 3D-Wind algorithm retrieves AMVs that correlate with operational GOES and MISR products and that MISR along-track winds are improved by the extra information provided from GOES that helps resolve the ambiguity between along-track cloud motion and parallax height. The analysis of retrieved heights and velocities for tracked ground features demonstrates the accuracy of the height assignments and when geometric heights are converted to pressure heights there is almost certainly an improvement relative to pressure heights assigned by IR methods. Using additional MISR cameras beyond the A cameras seems to have only a modest impact on the retrievals unless the horizontal constraint on AMVs is relaxed (merits further investigation).

Validation of height assignments against LiDAR measurements was not performed in this study. Such comparisons can be problematic for a number of reasons. First, LiDAR instruments such as CALIOP profile cloud structures only in a curtain along the orbital path. The swath width of a LEO-GEO combination such as MISR with GOES is also relatively narrow $(\sim 350 \mathrm{~km})$ and so comparisons can be made only when their ground tracks near-simultaneously cross. Unfortunately, MISR and CALIOP are not part of the same orbital train. The comparison between GEO-GEO wind/cloud heights and LiDAR is much easier as GEO satellites repetitively cover whole hemispheres and there are frequent opportunities to collocate observations with LiDAR. LiDAR-stereo comparisons are certainly interesting when they can be done, and as a second point, it should be recognized that each type of observation is measuring something a little different. MISR and GOES 3D Winds are the result of pattern matching using extended templates and therefore measure the height of the pattern in aggregate rather than the height of the LiDAR return from a small spot. CALIOP would then be sampling a thin track $\sim 100 \mathrm{~m}$ across a pattern template $>10 \mathrm{~km}$ that should not necessarily be expected 
to be uniform at the LiDAR scale. A third point is that the optical depth associated with the contrast for the pattern (estimated $\sim 0.3$ [57]) should be typically larger than that of the first LiDAR return; therefore, LiDAR assignments of cloud heights should be biased high with respect to stereo methods, which can be seen in Figure 2.

\section{Discussions}

We now consider the meaning of a wind height assignment and implications for future work, including low-cost systems for 3D-Wind retrievals that take advantage of the techniques just described.

\subsection{Stereo Height vs. IR Height}

Stereo height is perhaps the most consistent and accurate vertical registration of a cloud pattern since both stereo height and AMV can use the same pattern matcher to determine the feature's disparities in spatially and temporally separated images. A caveat would be that an AMV can be determined from two nadir images, whereas stereo height needs the nadir and slant views. Cloud features are assumed to be similar from different viewing angles, which should be valid for the majority of cloudy scenes. However, this assumption can break down sometimes in multi-layer cloud situations where the upper level cloud is transparent at the nadir view but opaque at the slant view.

To better understand the connection between the stereo height and the IR height derived from brightness temperature, we carry out a set of radiative transfer calculations from simulated cloud fields. We use the Joint Center for Satellite Data Assimilation (JCSDA) community radiative transfer model (CRTM) to compute both cloudy and clear-sky radiances at the top-of-atmosphere (TOA) at the $11 \mu \mathrm{m}$ band. CRTM includes scattering calculations for cloud, aerosol, gas molecular and the surface as specified, and has been the key backbone in DA systems [58].

In the simulation we use a cloudy atmosphere generated by a 3D cloud-resolving model (CRM) in a $512 \times 512 \mathrm{~km}$ region over the Eastern Pacific that includes cloud ice, liquid, snow, rain, graupel, and meteorological variables (e.g., temperature, pressure, and humidity). The simulated atmosphere has $1 \mathrm{~km}$ resolution with a realistic synoptic forcing from MERRA-2 reanalysis data [59]. The CRTM was used to produce the $11 \mu \mathrm{m}$ radiances at the TOA for two viewing angles, $0^{\circ}$ and $45^{\circ}$, as shown in Figure 18. The simulated 3-D cloud variables are first interpolated to a vertical grid with $250 \mathrm{~m}$ spacing, to match the horizontal resolution $(1 \mathrm{~km})$ in a $1: 4$ ratio. For the $45^{\circ}$ slant path calculation, we used the staggered shift method that was employed before in Gong et al. [60] and Várnai and Davies [61], to select the cloud profile indices and pick every 4th vertical index for each horizontal index shift. These staggered shift profiles, starting from the model top ( $25 \mathrm{~km})$ to the surface, are input to CRTM to create the cloud images at $45^{\circ}$ view. The $45^{\circ}$ cloud image is further used for pattern matching with the nadir-view image simulated using a vertical profile at every 4 th vertical index. All the simulated images have effective vertical and horizontal resolutions of $1 \mathrm{~km}$. CRTM's K-matrix mode outputs the Jacobians of the simulated radiances with respect to atmospheric and cloud variable.

A $20 \times 20-\mathrm{km}$ domain is used for stereo pattern matching, and the retrieved stereo height is compared with cloud heights from various definitions. Figure 19 compares the stereo height with three geometric CTHs: domain average, domain maximum, and domain top-20\% average. The domain-mean CTHs are consistently lower than the stereo heights except for a few high clouds, while domain-max and domain-top-20\% average are generally higher except for some low clouds. Thus, cloud geometric top heights are not well associated with the 11- $\mu \mathrm{m}$ stereo height. 
(a)
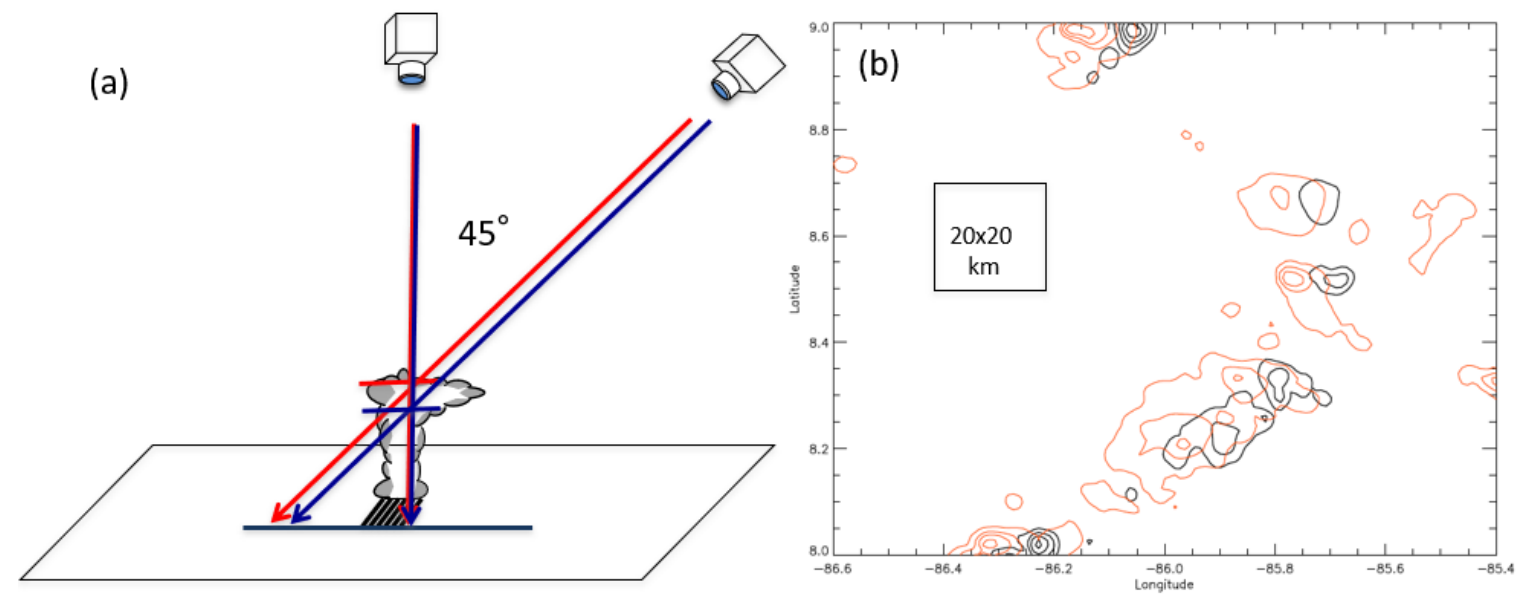

Figure 18. (a) Viewing geometry for a simulated cloud; (b) Simulated $11 \mu \mathrm{m}$ radiances (in contours) from the $0^{\circ}$ (black) and $45^{\circ}$ (red) view angles, showing the parallax effect. Depending on the penetration depth of the imager wavelength, stereo height may correspond to a different cloud height, as indicated by the colored lines in (a); e.g., visible (blue) and IR (red).

Domain Mean

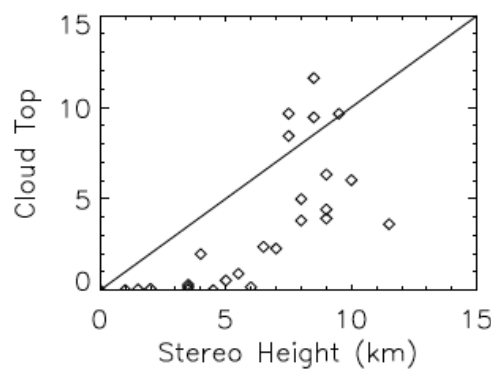

Domain Max

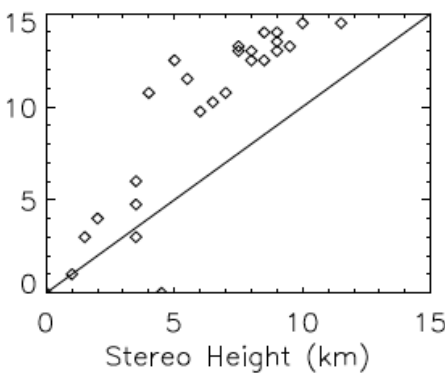

Domain Top20 Mean

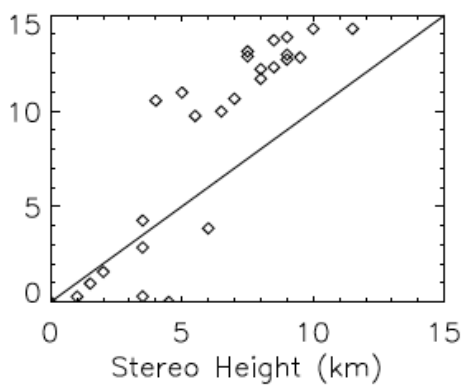

Figure 19. Comparisons of the stereo height with domain-mean, domain-maximum, and domain-top-20\% average CTHs.

What cloud properties are more closely associated with the 11- $\mu \mathrm{m}$ stereo height? Because the simulated data have all key cloud physical properties, we derive two weighted heights based on cloud internal properties: one weighted by vertical cloud mass profile (i.e., from ice, water, rain, graupel, snow) and the other weighted by the Jacobians with respective these cloud species. As shown in Figure 20, the $11-\mu \mathrm{m}$ stereo height is better represented by the domain maximum of the mass-weighted cloud height.
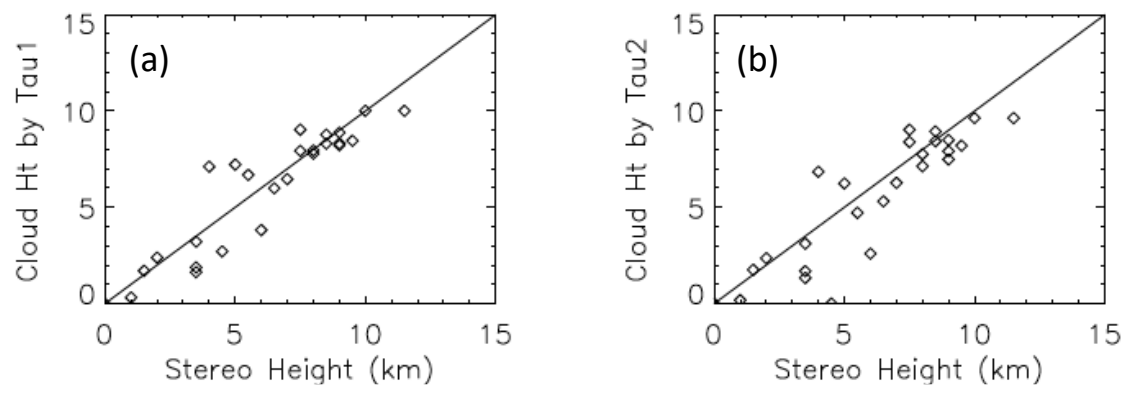

Figure 20. Comparisons of the stereo height with the domain maximum of two weighted cloud heights: (a) mass-weighted (Tau1) and (b) Jacobian-weighted (Tau2) cloud heights. 
In summary, the stereo height determined from IR channels is closely related to cloud microphysical properties and mass profile. Our simulations indicate that the IR stereo height would be representative of the cloud mass-weighted height. As illustrated in Figure 18a, differences in cloud penetration depth at different wavelengths should be expected to lead to different parallaxes and therefore different stereo heights. This is part of the fundamental problem with the IR method for height assignments of cloud patterns. As discussed earlier for semi-transparent clouds, the height of visible cloud patterns can be significantly different from the height that the IR method represents. A future study will need to evaluate the relationship between the stereo height from visible images and cloud properties. It is a fundamental challenge to understand the relationships between stereo heights, IR and visible radiances, and cloud microphysics, and an especially challenging problem to understand from first principles these relationships when considering visible stereo heights and cloud IR properties.

\subsection{Future Global AMVs with CubeSat Constellations}

The significantly improved AMV and height retrievals from MISR+GOES demonstrate the power of multi-platform wind observations for atmospheric research and future operational weather monitoring. Multi-platform stereo techniques can be applied across a constellation of operational GEO satellites and in LEO-GEO combinations. Figure 21 presents a concept for a constellation of multi-angle imaging systems hosted on CubeSats that are now available at a relatively low cost. This concept is similar to some previous proposals for similar observing systems [62-65]. Coupling LEO CubeSat observations with operational GEO satellites (i.e., GOES, Himawari, and Meteosat) has the benefit of both providing an additional vantage point and serving as a standard reference for geo-registration that can be used to calibrate registration biases and enable accurate 3D retrievals. High performance INR is not necessarily required of all components of the LEO-GEO combination since, as we have shown, systematic errors can be calibrated and compensated using the bundle-adjustment technique. Neither is synchronization of observations required between platforms as there is none between MISR and GOES. These observations point to the feasibility of deploying compact imaging systems on low-cost CubeSats. High-precision absolute pointing is not required as long as errors remain correlated over the collection time.

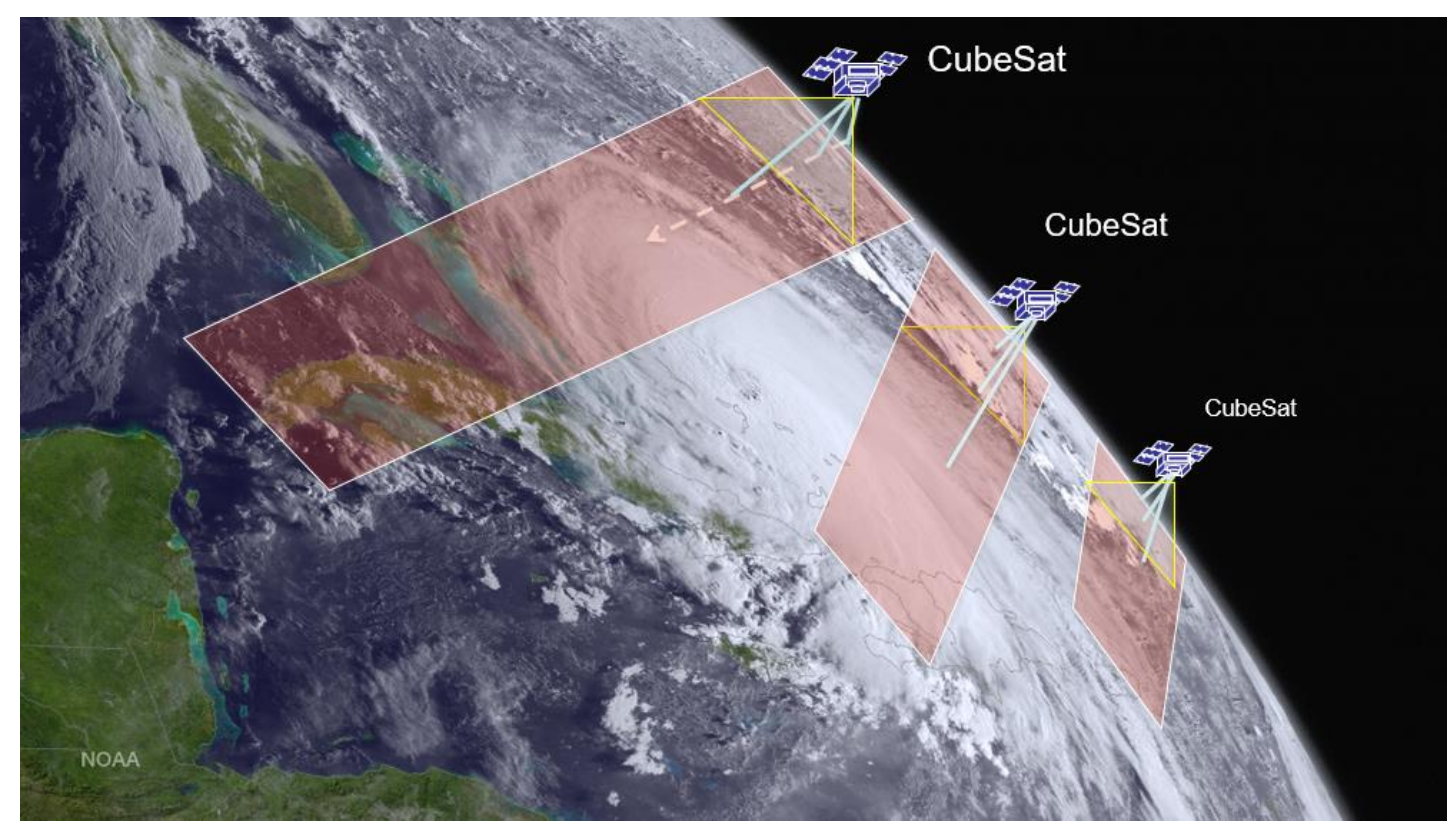

Figure 21. Illustration of CubeSat constellation under GOES images to provide multiple LEO-GEO pairs for 3D AMV and height retrievals. 
It is also possible to derive 3D AMVs using two LEO platforms. A concept for such a system is shown in Figure 22 with two CubeSats flying in loose formation. Imagery collected at three angles from each CubeSat provides information similar to that which MISR provides and successive nadir looks from each satellite provides analogous information to that provided by GOES, which enable full retrieval of both AMV velocities and heights. The high pixel resolution and multiple sampling along track from the LEO-LEO constellation can also provide vertical wind information as the horizontal winds and cloud height are precisely constrained.

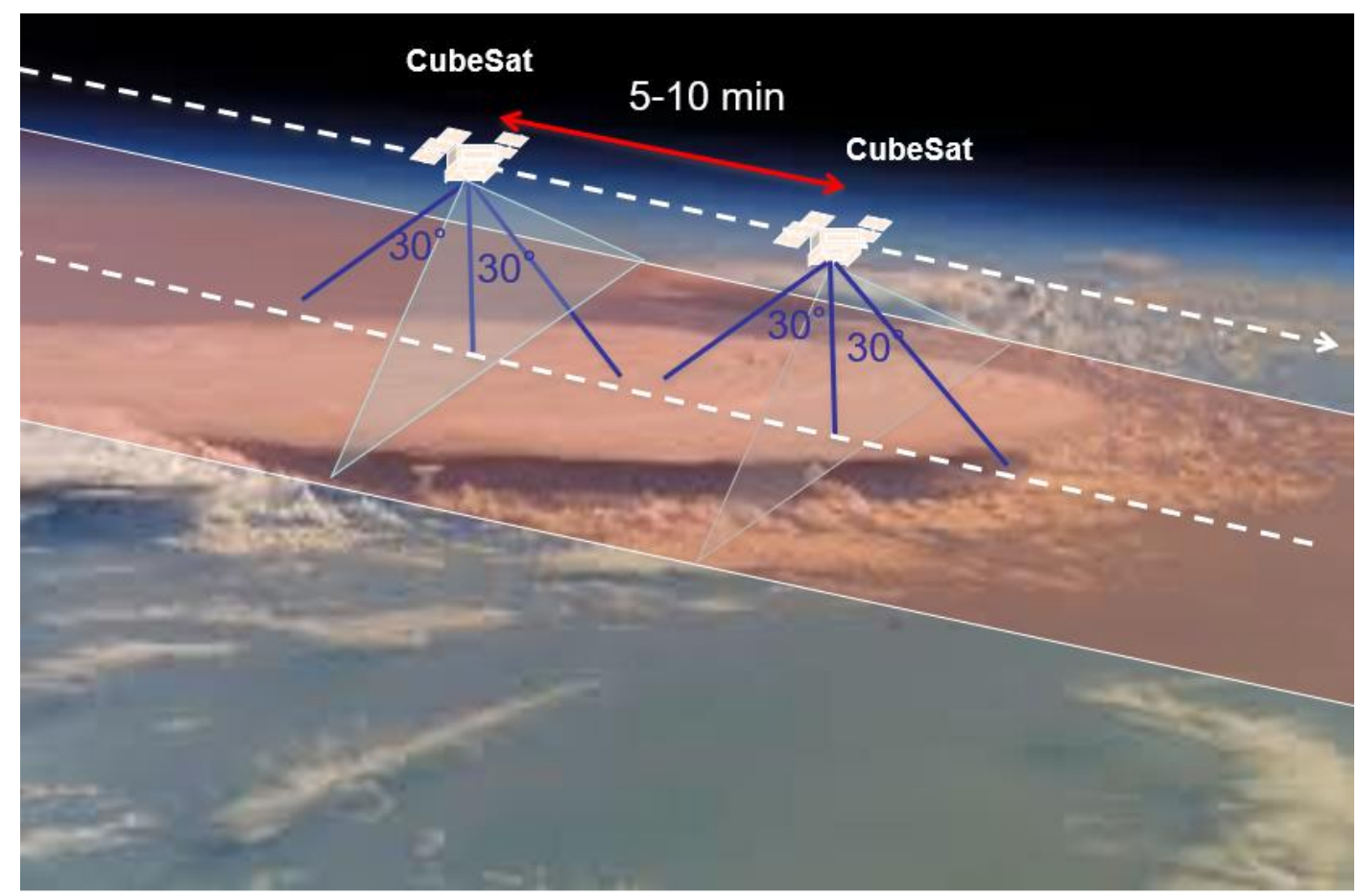

Figure 22. Illustration of two CubeSat formation flight to produce 3D AMV and height measurements along a single orbit.

\subsection{Compact Midwave Imaging System (CMIS)}

The great potential for CubeSat cloud imaging provides a strong motivation for developing the Compact Midwave Imaging System (CMIS). CMIS is an ongoing project funded by NASA's Earth Science Technology Office to advance the technical readiness level of a low size, weight, power and cost (SWaP-C) imager for a LEO mission to retrieve 3D-Wind AMVs and heights.

The CMIS instrument consists of three bands at 2.25, 3.75, and $4.05 \mu \mathrm{m}$ for sensing clouds, aerosols, and atmospheric particulates. The 3.75- $\mu \mathrm{m}$ channel provides aft, nadir, and fore views for stereo sensing. CMIS employs push broom imaging with a butcher block stripe filter installed immediately above the Type-2 Superlattice focal plane array (FPA) [66]. This type of detector operates at relatively high temperatures $(150 \mathrm{~K})$, while permitting CMIS to approach the sensitivity and radiometric performance of exquisite instruments such as ABI, MODIS and VIIRS. The system avoids the use of scanning mechanisms and requires only modest cooling to maintain the detector temperature at $150 \mathrm{~K}$, which keeps the instrument volume small and power draw low. The SWaP-C of CMIS is compatible with CubeSats and therefore offers the potential of a CubeSat constellation in LEO to provide high-quality, AMVs using the aforementioned techniques to provide rapidly revisited, global-scale observations. Such a constellation could provide good coverage at the poles provided that at least a few of the satellites are in highly inclined orbits.

The wavelength bands chosen for CMIS in the shortwave/mid-wave infrared (SWIR/MWIR) provide several distinct advantages. The Earth's MWIR radiance includes roughly equal contributions 
from solar reflected and terrestrial thermally emitted radiation. In the absence of sunlight, the MWIR band has sufficient signal to measure terrestrial features at night, while during the day it can leverage the differences between thermal emitted and solar reflected illumination. As shown in Figure 23, cloud features are clearly evident at night after the visible band loses sensitivity. The MWIR bands allow CMIS not only to provide full day/night coverage, but also to provide day/night stereo measurements for accurate height assignment derived from current GEO and LEO platforms that have a similar MWIR channel. Since the index of refraction is fairly uniform between 3.75 and $4.0 \mu \mathrm{m}$, the exact spectral bands of different imagers on GOES, Himawari and Meteosat should not have a significant impact on the pattern matching. An important objective of the CMIS development effort will be to adapt and prove current pattern matching algorithm in the MWIR to CMIS.

The ground sample distance (GSD) of the CMIS imagery will be between $600 \mathrm{~m}$ and $1.1 \mathrm{~km}$ depending on the specific altitude chosen for the mission. The GOES-R, Himawari, and Meteosat Third Generation imagers all have MWIR bands with $2 \mathrm{~km}$ resolution, so resolution ratios will be similar to that between the MISR red channel $(275 \mathrm{~m})$ and GOES-R B02 (500 m). Since the AMVs are sampled at coarser scales (2.2 $\mathrm{km}$ for this paper), the horizontal resolution of the motion vectors derived from CMIS should be comparable to those achieved for GOES+MISR.

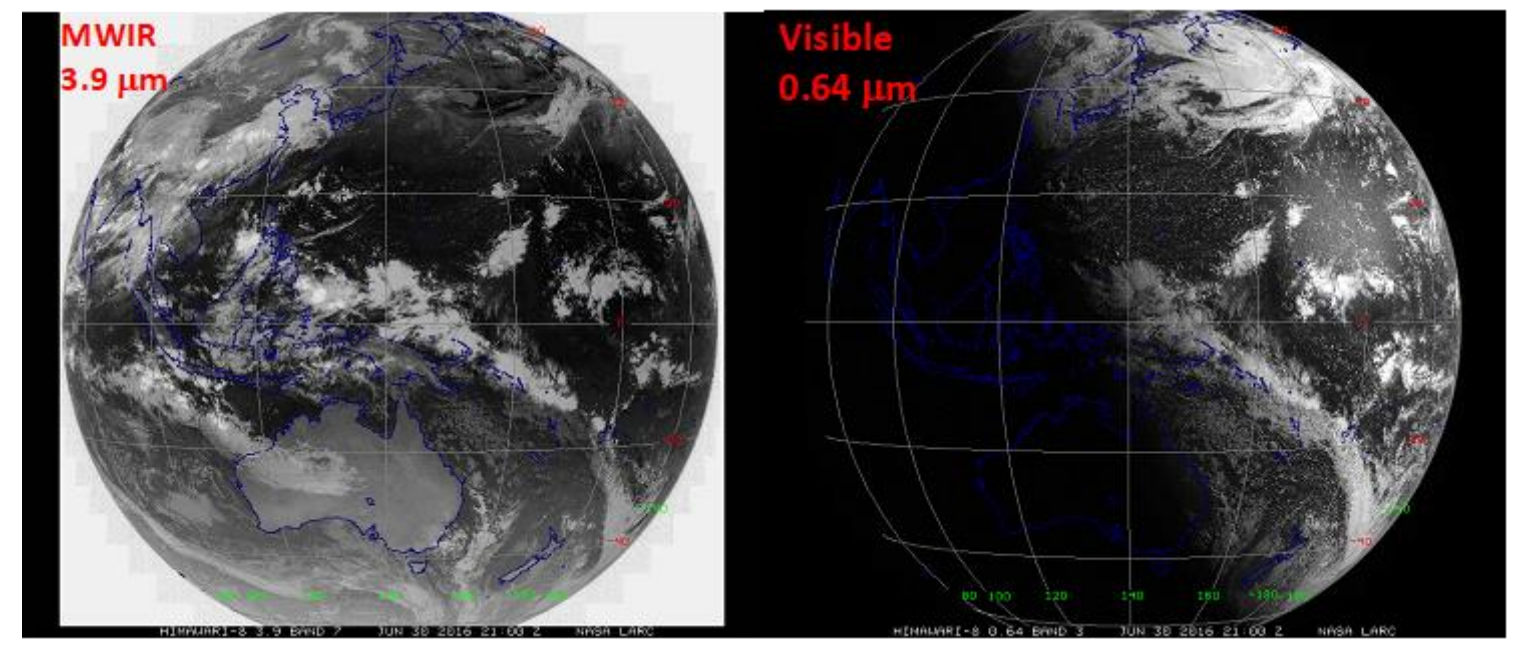

Figure 23. Himawari-8 MWIR $(3.9 \mu \mathrm{m})$ and visibile $(0.64 \mu \mathrm{m})$ at 21 UTC on 30 June 2016 show the extended cloud images at night from the MWIR band.

Airborne test flights scheduled in 2019 will be used to evaluate the performance of CMIS. The airborne test campaign will include four flights on a Gulfstream-IV at an altitude of $13.7 \mathrm{~km}$. JHU / APL plans to fly CMIS, plus two additional imagers with bands in the visible and longwave to allow for inter-comparison between height assignments based on the radiometric and stereo methods. The flights will be planned to under-fly GOES-16 and JPSS to enable testing of the multi-satellite stereo methodology described here to retrieve AMVs.

Given the low communications bandwidth typical of low SWaP-C platforms, onboard processing will likely be important for hosting CMIS on a CubeSat in order to compress the science data and apply the necessary metadata to time-tag and navigate the collected imagery. Some preliminary risk reduction work has been done at NASA Goddard Space Flight Center to prepare by implementing computer vision algorithms in a Field Programmable Gate Array (FPGA) that consumes very little power. This approach will allow the forward and aft MWIR camera looks to be transformed onboard into disparity maps such as is shown in Figure 24 and is an effective means of data compression. Deriving the fore/aft disparity with respect to the nadir look is the first step in the 3D-Wind algorithm that will be completed on the ground with complementary GEO data. 


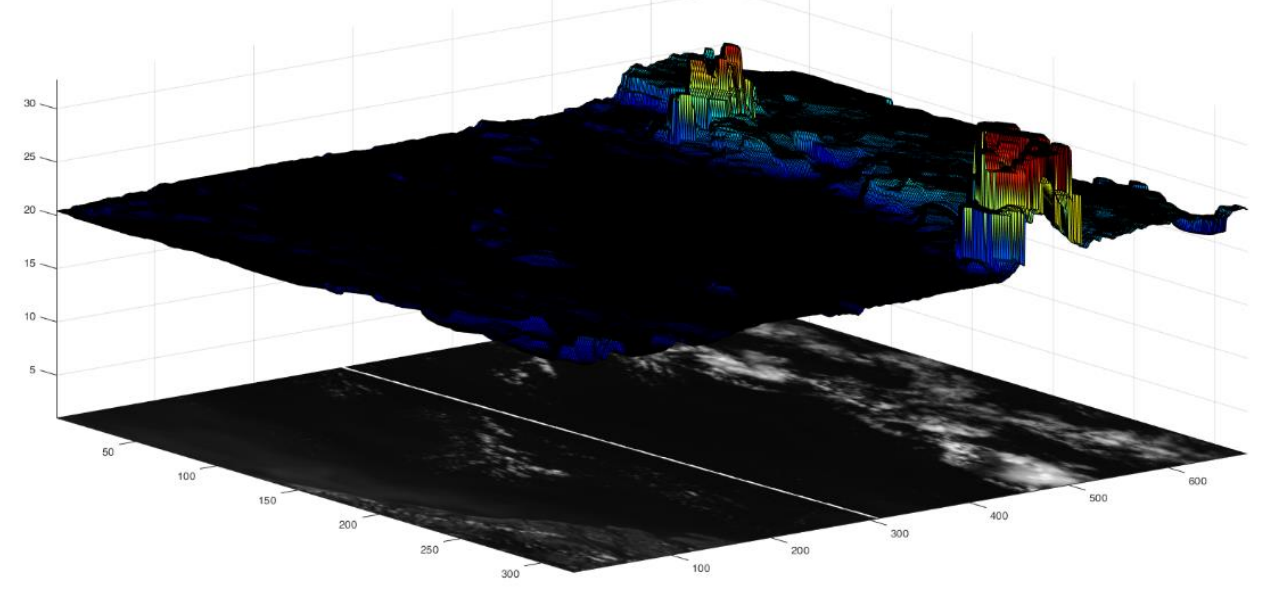

Figure 24. An example of disparities calculated in a test of an FPGA implementation suitable for a CubeSat mission. The implementation of Hirschmuller's algorithm [67] in the SpaceCube 2.0 processor [68] will be tested onboard the International Space Station in late 2019.

\section{Conclusions}

We have shown that accurate 3D-Wind retrievals can be made from multiple platforms using stereo imaging even if their spatial sampling is different, without synchronization of observations, and in the presence of systematic errors in the navigation of pixels to a reference ellipsoid. In this paper, the new algorithm was demonstrated for the LEO-GEO case and validated using the collocated MISR and GOES-16/17 datasets. The retrieved AMVs and heights over clear-sky terrain show that the retrievals are accurate to $<200 \mathrm{~m}$ in height and $<0.5 \mathrm{~m} / \mathrm{s}$ in velocity with a horizontal sampling of $2.2 \mathrm{~km}$. Because AMVs and stereo heights use the same pattern matching method, we argued and showed with terrain height retrievals that the stereo height derived from the 3D-Wind algorithm is a more representative and accurate height assignment for AMVs in comparison with the height assignments that use conventional thermal IR methods. The latter are subject to substantial uncertainties in the presence of weak or reversed vertical gradients in the atmospheric temperature profile such as in the planetary boundary layer (PBL) and in the case of tropopause folding. The height error in such cases is on the order of the PBL or folding thickness. Stereo imaging overcomes the challenge of the height assignment problem by providing additional and direct information on feature height in the form of geometric parallax. One must, of course, be careful with the meaning of "cloud height" as it relates to feature-track winds as we have demonstrated through modeling, and "pattern height" is perhaps a more accurate term. The problem of establishing the relationship between cloud and wind height also exists with IR methods for height assignment and perhaps more so when mixing information from visible and IR bands and in the case of multiple cloud layers.

The multi-angle imaging capability of MISR is very beneficial for stereo-height determinations. From its nine angular views in nadir, fore-, and aft-looking directions, MISR can independently determine the height and the cross-track component of wind, but the along-track wind and height are coupled and difficult to separate accurately. We have demonstrated that the extra geometric information provided by the vantage point of a GEO satellite uncouples the two and enables an improvement of the MISR-only wind product. Conversely, the LEO vantage point, especially in the case of multi-angle imaging, provides extra information that improves a GEO-only wind product. Systematic errors in the geographic registration of imagery from one platform to the other can be effectively compensated by the bundle-adjustment method, but it is important that they be correlated over the time that all observations are collected for a retrieval.

The 3D-Wind algorithm developed in this study is applicable in general to GEO-GEO, LEO-GEO, and LEO-LEO combinations, using Himawari, MSG, MODIS, or VIIRS, for greater spatiotemporal 
coverage. The technique demonstrated here using MISR and GOES has important implications for future global wind observations where high-quality AMV and height retrievals can be achieved with a low-cost constellation of CubeSat imaging sensors. We described one approach to such a capability using the CMIS instrument for multi-angle imaging from LEO either alone or in LEO-LEO pairs and an operational GEO environmental satellite.

Supplementary Materials: The following are available online at http:/ / www.mdpi.com/2072-4292/10/12/1885/ s1, Figure 2, Figure 3, Figure 4b, Figure 9, Figure 10, Figure 11, Figure 13b, Figure 16 as Matlab fig-files and P012O99670 and P024O098797 (with W-component AMVs) 3D-Winds products as a netCDF file.

Author Contributions: Conceptualization, J.L.C., D.L.W., M.A.K. and J.G.; Data curation, J.L.C.; Formal analysis, J.L.C. and D.L.W.; Funding acquisition, D.L.W. and M.A.K.; Investigation, J.L.C., D.L.W. and J.G.; Methodology, J.L.C. and D.L.W.; Project administration, D.L.W.; Resources, D.L.W.; Software, J.L.C.; Supervision, D.L.W.; Validation, J.L.C. and D.L.W.; Visualization, J.L.C. and M.A.K.; Writing-original draft, J.L.C., D.L.W. and M.A.K.; Writing-review \& editing, J.L.C., D.L.W. and J.G.

Funding: Carr Astronautics was supported through a task on the Support for Atmospheres, Modeling, and Data Assimilation (SAMDA) contact at NASA Goddard Space Flight Center (NNG17HP01C), prime contractor, Science Systems and Applications, Inc. (SSAI).

Acknowledgments: The MISR data were obtained from the NASA Langley Research Center Atmospheric Science Data Center. The GOES-16 data were obtained from the NOAA Comprehensive Large Array-data Stewardship System (CLASS). GOES-17 preliminary, non-operational data is courtesy of Vladimir Kondratovich of Earth Resources Technology (ERT). This study was supported by NASA's Terra/MISR and ESTO/IIP programs. We thank David Liu of SSAI for providing the MERRA2 data and Ákos Horváth for providing METEOSAT-10 AMV height statistics. Houria Madani, Norman Lo, and Fangyuan Ding of Carr Astronautics have supported our 3D-Winds IR\&D project. Houria Madani provided the CALIPSO comparisons (contributing Figure 2), helped develop the theory for the original multi-GEO work cited here, and coauthored much of our original multi-GEO 3D-Winds code. Norman Lo developed the FPGA-assisted computer vision code for the SpaceCube 2.0 hardware with support from the NASA Goddard Space Flight Center under the Omnibus Multidisciplinary Engineering Services-II (OMES-II) contract and contributed Figure 24. Fangyuan Ding adapted the first version of our multi-GEO 3D-Winds code to work with full-disk imagery and contributed Figure 3. Special thanks to Stacey Williamson at Carr Astronautics who assisted with the bibliography and final production of the manuscript.

Conflicts of Interest: The authors declare no conflict of interest.

\section{Appendix A MISR-GOES 3D-Wind Algorithm Math Model}

The math model of the 3D-Wind algorithm for joint MISR and GOES 3D-Wind retrievals is described here. MISR imagery from its fore, aft and nadir cameras are used along with GOES Full Disk (FD), CONUS, or Mesoscale (MESO) imagery from $T_{0}-\Delta T, T_{0}$, and $T_{0}+\Delta T$, where $T_{0}$ is a GOES product time closest to the time of the selected MISR nadir-camera data block and $\Delta T$ is the GOES product refresh period. The MISR dataset used has already been rectified to the WGS84 ellipsoid and mapped into a Space Oblique Mercator (SOM) projection. The GOES dataset has also already been rectified to the GOES fixed-grid which is similarly a projection over the WGS84 ellipsoid. Using the MISR An camera as a common reference, we measure disparities in the MISR fore and aft cameras and the triplet of GOES images. Each disparity locates an object $\mathcal{O}$, represented by a template centered at a tie point in the MISR An camera image, in any of the five (or more) other images. From the disparities, we calculate the geographic coordinates for each object $\mathcal{O}$ as it would appear if it were to rest on the ellipsoid. The lack of simultaneity between images means the motion of $\mathcal{O}$ must be explicitly formulated in the model, which is obviously necessary for wind vector estimation but is confounding for parallax height assignments.

\section{A.1. Formulation of the Problem.}

First suppose a static object $\mathcal{O}$ and let $\vec{r}_{n}(\mathcal{O})$ be where it appears to rest on the ellipsoid as viewed from satellite/look $n$. This vector would be calculated from the SOM coordinates as provided in the MISR product. Reserve $n=0$ to designate the MISR nadir camera from which we draw the template. The true position vector for the object $\mathcal{O}$ would be $\vec{r}(\mathcal{O})=\vec{r}_{0}(\mathcal{O})+\vec{\delta}$, where $\vec{\delta}$ is the adjustment needed to position $\mathcal{O}$ in 3D space off the ellipsoid. We seek to discover $\vec{\delta}$ for which the component in 
the direction of the local vertical would be the height assignment. Figure A1 shows the geometry of the problem to be solved. The tangent plane is constructed at the point on the ellipsoid where look $n \neq 0$ assigns the object $\mathcal{O}$.

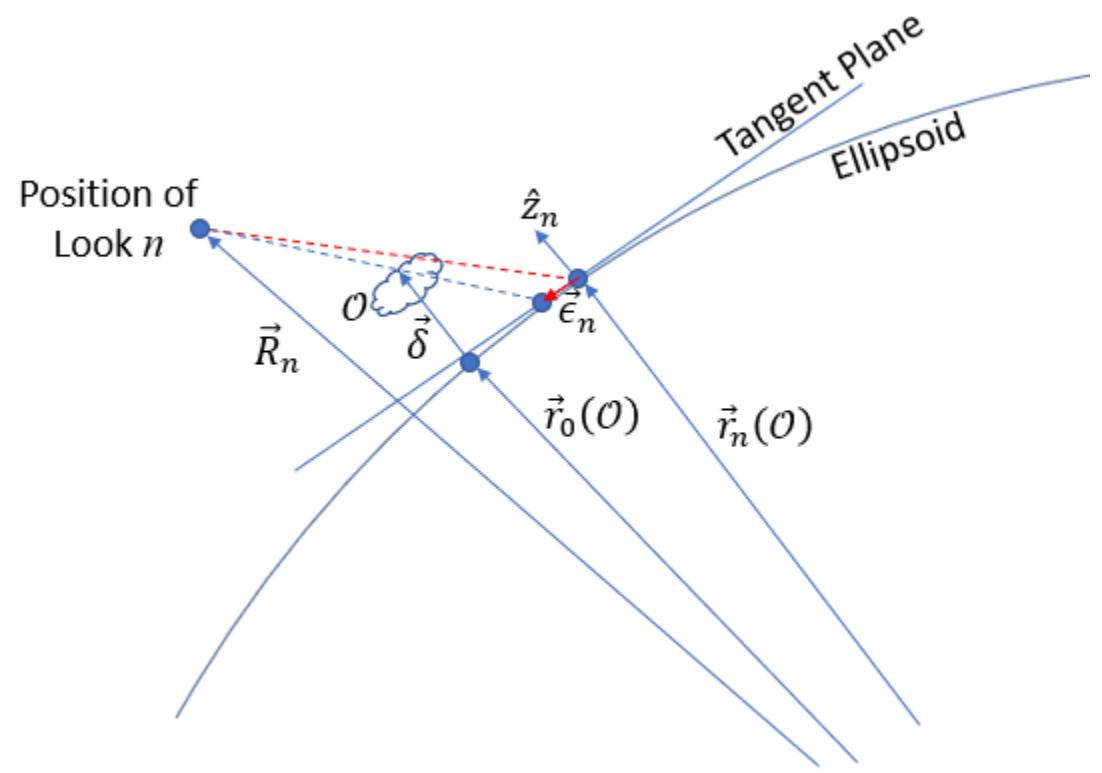

Figure A1. Problem geometry showing looks $n \neq 0$ and $n=0$.

Consider a single disparity pairing for which the matching algorithm has found the SOM coordinates for $\mathcal{O}$ as it would appear from satellite/look $n$ were it to sit on the ellipsoid. The line-of-sight to $\mathcal{O}$ for satellite/look $n$ starts at the position vector for the satellite $\left(\vec{R}_{n}\right)$ and runs parallel to the vector touching the apparent location of $\mathcal{O}$ on the ellipsoid $\left(\vec{r}_{n}(\mathcal{O})\right)$ and must run through the 3D location of the object $\vec{r}(\mathcal{O})=\vec{r}_{0}(\mathcal{O})+\vec{\delta}$. Expressed mathematically, there must be some multiplier $\lambda^{-1}$ that makes this equation hold:

$$
\vec{r}_{0}(\mathcal{O})+\vec{\delta}=\lambda^{-1}\left(\vec{r}_{n}(\mathcal{O})-\vec{R}_{n}+\vec{\epsilon}_{n}\right)+\vec{R}_{n}
$$

The apparent position of $\mathcal{O}$ on the ellipsoid $\left(\vec{r}_{n}(\mathcal{O})\right)$ could not have been determined perfectly, so we have introduced a vector $\vec{\epsilon}_{n}$ in Equation (A1) to make equality hold that we can interpret as deriving from an error or uncertainty in the disparity measurement. Normally, $\vec{\epsilon}_{n}$ should be small even if the disparity is large. All imagery has been mapped into SOM coordinates, the point of correspondence found where the template best matches, the SOM coordinates of the match inverted to determine the latitude and longitude on the ellipsoid, and finally the apparent position vector $\left(\vec{r}_{n}(\mathcal{O})\right)$ calculated. In doing so, $\vec{r}_{n}(\mathcal{O})$ can only rest on the ellipsoid, so we can consider small deviations in the calculations represented by $\vec{\epsilon}_{n}$ as sitting in the tangent plane at $\vec{r}_{n}(\mathcal{O})$; i.e., $\hat{z}_{n} \cdot \vec{\epsilon}_{n}=0$, where $\hat{z}_{n}$ is the local vertical of the ellipsoid at $\vec{r}_{n}(\mathcal{O})$. We rearrange Equation (A1) to isolate $\vec{\epsilon}_{n}$ on the left-hand side:

$$
\vec{\epsilon}_{n}=\lambda\left(\vec{r}_{0}(\mathcal{O})-\vec{R}_{n}+\vec{\delta}\right)-\left(\vec{r}_{n}(\mathcal{O})-\vec{R}_{n}\right) .
$$

Equation (A2) is used to derive an equation for $\lambda$ from the constraint $\hat{z}_{n} \cdot \vec{\epsilon}_{n}=0$,

$$
\lambda=\frac{\hat{z}_{n} \cdot\left(\vec{r}_{n}(\mathcal{O})-\vec{R}_{n}\right)}{\hat{z}_{n} \cdot\left(\vec{r}_{0}(\mathcal{O})-\vec{R}_{n}+\vec{\delta}\right)} .
$$


Equation (A3) is nonlinear in $\vec{\delta}$, but given a guess $\vec{\delta}_{0}$ for the value of $\vec{\delta}$, we can linearize it in a Taylor series:

$$
\lambda \cong \frac{\hat{z}_{n} \cdot\left(\vec{r}_{n}(\mathcal{O})-\vec{R}_{n}\right)}{\hat{z}_{n} \cdot\left(\vec{r}_{0}(\mathcal{O})-\vec{R}_{n}+\vec{\delta}_{0}\right)}-\frac{\hat{z}_{n} \cdot\left(\vec{r}_{n}(\mathcal{O})-\vec{R}_{n}\right)}{\left(\hat{z}_{n} \cdot\left(\vec{r}_{0}(\mathcal{O})-\vec{R}_{n}+\vec{\delta}_{0}\right)\right)^{2}} \hat{z}_{n} \cdot\left(\vec{\delta}-\vec{\delta}_{0}\right)
$$

Substituting $\lambda$ from Equation (A4) into (A2) and discarding terms higher than linear in $\vec{\delta}-\vec{\delta}_{0}$ gives an approximate linear measurement model:

$$
\vec{\epsilon}_{n}=A_{n}\left(\vec{\delta}_{0}\right) \cdot\left(\vec{\delta}-\vec{\delta}_{0}\right)-\vec{b}_{n}\left(\vec{\delta}_{0}\right)
$$

The matrix $A_{n}\left(\vec{\delta}_{0}\right)$ and vector $\vec{b}_{n}\left(\vec{\delta}_{0}\right)$ are defined accordingly (with $I$ as the identity matrix):

$$
\begin{gathered}
A_{n}\left(\vec{\delta}_{0}\right)=\lambda_{0}\left[I-\frac{\left(\vec{r}_{0}(\mathcal{O})-\vec{R}_{n}+\vec{\delta}_{0}\right) \hat{z}_{n}{ }^{T}}{\hat{z}_{n} \cdot\left(\vec{r}_{0}(\mathcal{O})-\vec{R}_{n}+\vec{\delta}_{0}\right)}\right], \\
\vec{b}_{n}\left(\vec{\delta}_{0}\right)=\left(\vec{r}_{n}(\mathcal{O})-\vec{R}_{n}\right)-\lambda_{0}\left(\vec{r}_{0}(\mathcal{O})-\vec{R}_{n}+\vec{\delta}_{0}\right) . \\
\lambda_{0}=\frac{\hat{z}_{n} \cdot\left(\vec{r}_{n}(\mathcal{O})-\vec{R}_{n}\right)}{\hat{z}_{n} \cdot\left(\vec{r}_{0}(\mathcal{O})-\vec{R}_{n}+\vec{\delta}_{0}\right)} .
\end{gathered}
$$

\section{A.2. Solution to the Static Problem.}

Equation (A5) is effectively only two equations because its projection along $\hat{z}_{n}$ vanishes by construction, so an additional look is required to solve for the three parameters represented by $\vec{\delta}$. Adding just one additional look makes the problem overdetermined; therefore, we can only solve for $\vec{\delta}$ in a least squares sense by minimizing

$$
\chi^{2}=\sum_{n=1}^{N} \vec{\epsilon}_{n}{ }^{T} W_{n} \vec{\epsilon}_{n}
$$

A weighting matrix $W_{n}$ has been introduced. It can be the identity matrix for an unweighted solution. This solution to this least-squares problem is the solution to the linear system:

$$
\left(\sum_{n=1}^{N} A_{n}\left(\vec{\delta}_{0}\right)^{T} W_{n} A_{n}\left(\vec{\delta}_{0}\right)\right)\left(\vec{\delta}-\vec{\delta}_{0}\right)=\sum_{n=1}^{N} A_{n}\left(\vec{\delta}_{0}\right)^{T} W_{n} \vec{b}_{n}\left(\vec{\delta}_{0}\right)
$$

To solve for $\vec{\delta}$ as a nonlinear problem, we start with a guess $\vec{\delta}_{0}$, solve for $\Delta \vec{\delta}=\vec{\delta}-\vec{\delta}_{0}$, update the guess and iterate. The solution is considered to have converged once $|\Delta \vec{\delta}|$ is less than a tolerance. If the measurement errors for the disparities are random and uncorrelated and each is weighted by a $W_{n}$ that is the inverse of the covariance matrix of its uncertainty, then the uncertainty of the estimate for $\vec{\delta}$ is described by a covariance matrix $P$ that is given by the coefficient matrix in Equation (A8):

$$
P=\left(\sum_{n=1}^{N} A_{n}\left(\vec{\delta}_{0}\right)^{T} W_{n} A_{n}\left(\vec{\delta}_{0}\right)\right)^{-1} .
$$

The square roots of the diagonal elements of $P$ can be interpreted as error bars for each of the solved for parameters in accordance with the statistical description of the disparity measurement errors. 


\section{A.3. Solution to the Dynamic Problem.}

Consider the dynamic problem where $\vec{\delta}$ is a function of time. We represent the time tag for $\mathcal{O}$ as $t_{n}(\mathcal{O})$ in satellite/look $n$ and assume steady velocity $\vec{v}$, so that

$$
\vec{\delta}(t)=\vec{\delta}_{0}+\vec{v} \cdot\left(t-t_{0}(\mathcal{O})\right)
$$

Equation (A5) is therefore generalized to

$$
\vec{\epsilon}_{n}=\left[\begin{array}{ll}
A_{n} & \left(t_{n}(\mathcal{O})-t_{0}(\mathcal{O})\right) A_{n}
\end{array}\right] X-\vec{b}_{n}
$$

with a state

$$
X=\left[\begin{array}{c}
\vec{\delta}_{0} \\
\vec{v}
\end{array}\right]
$$

Equation (A7) now has the solution, with $\Delta t_{n}=\left(t_{n}(\mathcal{O})-t_{0}(\mathcal{O})\right)$ :

$$
\sum_{n=1}^{N}\left[\begin{array}{cc}
A_{n}{ }^{T} W_{n} A_{n} & \Delta t_{n} A_{n}{ }^{T} W_{n} A_{n} \\
\Delta t_{n} A_{n}{ }^{T} W_{n} A_{n} & \left(\Delta t_{n}\right)^{2} A_{n}{ }^{T} W_{n} A_{n}
\end{array}\right] X=\sum_{n=1}^{N}\left[\begin{array}{c}
A_{n}{ }^{T} W_{n} \vec{b}_{n} \\
\Delta t_{n} A_{n}{ }^{T} W_{n} \vec{b}_{n}
\end{array}\right]
$$

The full 3D-wind retrieval is the solution of this six-dimensional linear system. Winds can be constrained to the tangent plane, in which case the state $X$ would have five states, three position adjustments $\vec{\delta}_{0}$ and two tangent-plane wind velocity components.

\section{A.4. Bundle Adjustment.}

Systematic errors may be seen in the disparity measurements because of small geo-registration errors. The important consideration is the relative error. If this is nearly constant and uniform for a short time across the MISR blocks, then the relative bias can be built into the retrieval process by introducing states to model it that will be estimated simultaneously with the 3D-wind retrieval states $X_{m}$ at each individual site $m$. This approach is called a bundle adjustment [69]. Suppose that six disparity measurements are made from the MISR An camera to the Aa, An (self), Af, and three GOES multitemporal images corresponding to indices $n=1, \ldots, 6$. We use all but the self $(n=2)$ case in the 3D-wind retrieval. The simplest model would be to represent the relative registration error between a block of MISR data and GOES as a fixed displacement in the tangent plane $\vec{c}$. Equation (A11) needs to change to include the new states $\vec{c}$ for $n=4,5,6$ :

$$
\vec{\epsilon}_{n, m}=\left[\begin{array}{lll}
A_{n, m} & \Delta t_{n} A_{n, m} & I
\end{array}\right]\left[\begin{array}{c}
X_{m} \\
\vec{c}
\end{array}\right]-\vec{b}_{n, m} .
$$

The estimation problem to minimize the weighted sum squared of all disparities with the full state $X^{T}=\left[\begin{array}{cccc}X_{1}{ }^{T} & \ldots & X_{M}{ }^{T} & \vec{c}^{T}\end{array}\right]$ can be effectively formulated and solved as a sparse matrix problem even if $M>10^{4}$.

\section{References}

1. Kelly, G.; Thépaut, J.-N.; Buizza, R.; Cardinali, C. The value of observations. I: Data denial experiments for the Atlantic and the Pacific. Q. J. R. Meteorol. Soc. 2007, 133, 1803-1815. [CrossRef]

2. Gelaro, R.; Langland, R.H.; Pellerin, S.; Todling, R. The THORPEX Observation Impact Intercomparison Experiment. Mon. Weather Rev. 2010, 138, 4009-4025. [CrossRef]

3. Gelaro, R.; Zhu, Y. Examination of observation impacts derived from observing system experiments (OSEs) and adjoint models. Tellus A Dyn. Meteorol. Oceanogr. 2016, 61, 179-193. [CrossRef] 
4. Menzel, W.P.; Frey, R.A.; Zhang, H.; Wylie, D.P.; Moeller, C.C.; Holz, R.E.; Maddux, B.; Baum, B.A.; Strabala, K.I.; Gumley, L.E. MODIS Global Cloud-Top Pressure and Amount Estimation: Algorithm Description and Results. J. Appl. Meteorol. Clim. 2008, 47, 1175-1198. [CrossRef]

5. Holz, R.E.; Ackerman, S.A.; Nagle, F.W.; Frey, R.; Dutcher, S.; Kuehn, R.E.; Vaughan, M.A.; Baum, B. Global Moderate Resolution Imaging Spectroradiometer (MODIS) cloud detection and height evaluation using CALIOP. J. Geophys. Res. Atmos. 2008, 113. [CrossRef]

6. Berthier, S.; Chazette, P.; Pelon, J.; Baum, B. Comparison of cloud statistics from spaceborne lidar systems. Atmos. Chem. Phys. 2008, 8, 6965-6977. [CrossRef]

7. Garay, M.J.; de Szoeke, S.P.; Moroney, C.M. Comparison of marine stratocumulus cloud top heights in the southeastern Pacific retrieved from satellites with coincident ship-based observations. J. Geophys. Res. 2008, 113. [CrossRef]

8. Schmetz, J.; Nuret, M. Automatic Tracking of High-Level Clouds in Meteosat Infrared Images with a Radiance Windowing Technique. ESA J. 1987, 11, 275-286.

9. Nieman, S.J.; Schmetz, J.; Menzel, W.P. A Comparison of Several Techniques to Assign Heights to Cloud Tracers. J. Appl. Meteorol. 1993, 32, 1559-1568. [CrossRef]

10. Menzel, W.P.; Smith, W.L.; Stewart, T.R. Improved Cloud Motion Wind Vector and Altitude Assignment Using VAS. J. Clim. Appl. Meteorol. 1983, 22, 377-384. [CrossRef]

11. Frey, R.A.; Baum, B.A.; Menzel, W.P.; Ackerman, S.A.; Moeller, C.C.; Spinhirne, J.D. A comparison of cloud top heights computed from airborne lidar and MAS radiance data using $\mathrm{CO}^{2}$ slicing. J. Geophys. Res. Atmos. 1999, 104, 24547-24555. [CrossRef]

12. Szejwach, G. Determination of Semi-Transparent Cirrus Cloud Temperature from Infrared Radiances: Application to METEOSAT. J. Appl. Meteorol. 1982, 21, 384-393. [CrossRef]

13. Schmetz, J.; Holmlund, K.; Hoffman, J.; Strauss, B.; Mason, B.; Gaertner, V.; Koch, A.; Berg, L.V.D. Operational Cloud-Motion Winds from Meteosat Infrared Images. J. Appl. Meteorol. 1993, 32, 1206-1225. [CrossRef]

14. Borde, R.; Oyama, R. A Direct Link Between Feature Tracking and Height Assignment of Operational Atmospheric Motion Vectors. In Proceedings of the 9th International Winds Workshop, Annapolis, MD, USA, 14-18 April 2008.

15. Borde, R.; Smet, A.D.; Arriaga, A. Height assignment of atmospheric motion vectors with Meteosat 8. In Proceedings of the Remote Sensing, Maspalomas, Spain, 30 November 2004; p. 9. [CrossRef]

16. Zhang, H.; Menzel, W.P. Improvement in thin cirrus retrievals using an emissivity-adjusted CO2 slicing algorithm. J. Geophys. Res. Atmos. 2002, 107. [CrossRef]

17. Velden, C.S.; Bedka, K.M. Identifying the Uncertainty in Determining Satellite-Derived Atmospheric Motion Vector Height Attribution. J. Appl. Meteorol. Clim. 2009, 48, 450-463. [CrossRef]

18. Lean, K.; Bormann, N.; Salonen, K. Assessment of new AMV data in the ECMWF system: First year report. EUMETSAT/ECMWF Fellowship Programme Research Report No. 43. 2017. Available online: https: / / www.ecmwf.int/sites/default/files/elibrary/2017/16984-assessment-new-amv-data-ecmwfsystem-first-year-report.pdf (accessed on 22 November 2018).

19. Whitehead, V.S.; Browne, I.D.; Garcia, J.G. Cloud height contouring from Apollo 6 photography. Bull. Am. Meteorol. Soc. 1969, 50, 522-529. [CrossRef]

20. Minzner, R.A.; Shenk, W.E.; Teagle, R.D.; Steranka, J. Stereographic cloud heights from imagery of SMS/GOES satellites. Geophys. Res. Lett. 1978, 5, 21-24. [CrossRef]

21. Hasler, A.F. Stereographic Observations from Geosynchronous Satellites: An Important New Tool for the Atmospheric Sciences. Bull. Am. Meteorol. Soc. 1981, 62, 194-212. [CrossRef]

22. Mack, R.; Hasler, A.F.; Rodgers, E.B. Stereoscopic observations of hurricanes and tornadic thunderstorms from geosynchronous satellites. Adv. Space Res. 1983, 2, 143-151. [CrossRef]

23. Takashima, T.; Takayama, Y.; Matsuura, K.; Naito, K. Cloud Height Determination by Satellite Stereography. Pap. Meteorol. Geophys. 1982, 33, 65-78. [CrossRef]

24. Fujita, T.T.; Dodge, J.C. Applications of stereoscopic height computations from dual geosynchronous satellite data/joint NASA-Japan stereo project. Adv. Space Res. 1983, 2, 153-160. [CrossRef]

25. Lancaster, R.S.; Spinhirne, J.D.; Manizade, K.F. Combined Infrared Stereo and Laser Ranging Cloud Measurements from Shuttle Mission STS-85. J. Atmos. Ocean. Technol. 2003, 20, 67-78. [CrossRef]

26. Horváth, Á.; Davies, R. Simultaneous retrieval of cloud motion and height from polar-orbiter multiangle measurements. Geophys. Res. Lett. 2001, 28, 2915-2918. [CrossRef] 
27. Zong, J.; Davies, R.; Muller, J.P.; Diner, D.J. Photogrammetric retrieval of cloud advection and top height from the multi-angle imaging spectroradiometer (MISR). Photogramm. Eng. Remote Sens. 2002, 68, 821-829.

28. Davies, R.; Horváth, Á.; Moroney, C.; Zhang, B.; Zhu, Y. Cloud motion vectors from MISR using sub-pixel enhancements. Remote Sens. Environ. 2007, 107, 194-199. [CrossRef]

29. Diner, D.J.; Braswell, B.H.; Davies, R.; Gobron, N.; Hu, J.; Jin, Y.; Kahn, R.A.; Knyazikhin, Y.; Loeb, N.; Muller, J.-P.; et al. The value of multiangle measurements for retrieving structurally and radiatively consistent properties of clouds, aerosols, and surfaces. Remote Sens. Environ. 2005, 97, 495-518. [CrossRef]

30. Mueller, K.J.; Wu, D.L.; Horváth, Á.; Jovanovic, V.M.; Muller, J.-P.; Girolamo, L.D.; Garay, M.J.; Diner, D.J.; Moroney, C.M.; Wanzong, S. Assessment of MISR Cloud Motion Vectors (CMVs) Relative to GOES and MODIS Atmospheric Motion Vectors (AMVs). J. Appl. Meteorol. Clim. 2017, 56, 555-572. [CrossRef]

31. Key, J.R.; Santek, D.; Velden, C.S.; Bormann, N.; Thepaut, J.; Riishojgaard, L.P.; Yanqiu, Z.; Menzel, W.P. Cloud-drift and water vapor winds in the polar regions from MODISIR. IEEE Trans. Geosci. Remote Sens. 2003, 41, 482-492. [CrossRef]

32. Dworak, R.; Key, J.R. Twenty Years of Polar Winds from AVHRR: Validation and Comparison with ERA-40. J. Appl. Meteorol. Clim. 2009, 48, 24-40. [CrossRef]

33. Santek, D. The Impact of Satellite-Derived Polar Winds on Lower-Latitude Forecasts. Mon. Weather Rev. 2010, 138, 123-139. [CrossRef]

34. Lazzara, M.A.; Coletti, A.; Diedrich, B.L. The possibilities of polar meteorology, environmental remote sensing, communications and space weather applications from Artificial Lagrange Orbit. Adv. Space Res. 2011, 48, 1880-1889. [CrossRef]

35. Santek, D.; Nebuda, S.; Stettner, D. Feature-tracked winds from moisture fields derived from AIRS sounding retrievals. In Proceedings of the 12th International Winds Workshop, Copenhagen, Denmark, 15-20 June 2014; p. 7655.

36. Hautecoeur, O.; Borde, R. Derivation of Wind Vectors from AVHRR/MetOp at EUMETSAT. J. Atmos. Ocean. Technol. 2017, 34, 1645-1659. [CrossRef]

37. Madani, H.; Carr, J.L. Stereo Cloud Top Height Products for the GOES-R Era. In Proceedings of the NOAA Satellite Conference, Greenbelt, Maryland, 27 April-1 May 2015.

38. Carr, J.L.; Gibbs, B.; Madani, H.; Allen, N. INR Performance of the GOES-13 Spacecraft Measured in Orbit. In Proceedings of the 31st Annual Rocky Mountain Guidance, Navigation and Control Conference, Breckenridge, CO, USA, 1-6 February 2008.

39. Gall, D.; Virgilio, V.; Forkert, R.; Van Naarden, J.; Griffith, P. GOES-16 ABI On-Orbit INR Tuning and Performance. Adv. Astronautical Sci. 2018, 164, 821. Available online: http:/ /www.univelt.com/linkedfiles/ v164\%20Contents.pdf (accessed on 22 November 2018).

40. Madani, H.; Carr, J.L.; Heidinger, A.; Wanzong, S. Inter-Comparisons between Radiometric and Geometric Cloud Top Height Products. Am. Geophys. Union 2015. Available online: https://agu.confex.com/agu/fm15/ mediafile/Handout/Paper76215/AGU_HouriaMadani_A33E_0220.pdf (accessed on 22 November 2018).

41. Carr, J.L. Multi-Satellite 3D Winds. NOAA Emerging Technologies Workshop; 2017. Available online: https:/ /nosc.noaa.gov/2017_NOAA_ETW/7_RFI_Session2/ (accessed on 22 November 2018).

42. Goes-R Series Product Definition and Users' Guide (PUG). 2018. Available online: https:/ / www.goes-r.gov/ users/docs/PUG-main-vol1.pdf (accessed on 22 November 2018).

43. Muller, J.; Mandanayake, A.; Moroney, C.; Davies, R.; Diner, D.J.; Paradise, S. MISR stereoscopic image matchers: Techniques and results. IEEE Trans. Geosci. Remote Sens. 2002, 40, 1547-1559. [CrossRef]

44. Mueller, K.; Moroney, C.; Jovanovic, V.; Garay, M.; Muller, J.; Girolamo, L.; Davies, R. (2013) MISR Level 2 cloud product algorithm theoretical basis. JPL Tech. Doc. 2013. Available online: https:/ / eospso.nasa.gov/ sites/default/files/atbd/MISR_L2_CLOUD_ATBD-1.pdf. (accessed on 22 November 2018).

45. Lewis, J.P. Fast Normalized Cross-Correlation. Industrial Light and Magic. 1995. Available online: https: //www.researchgate.net/publication/2378357_Fast_Normalized_Cross-Correlation (accessed on 22 November 2018).

46. Carr, J.L.; Moigne, J.; Netanyahu, N.; Eastman, R. Image Registration for Remote Sens; Cambridge University Press: Cambridge, UK, 2010.

47. Kalluri, S.; Alcala, C.; Carr, J.; Griffith, P.; Lebair, W.; Lindsey, D.; Race, R.; Wu, X.; Zierk, S. From Photons to Pixels: Processing Data from the Advanced Baseline Imager. Remote Sens. 2018, 10, 177. [CrossRef] 
48. Jovanovic, V.; Moroney, C.; Nelson, D. Multi-angle geometric processing for globally geo-located and co-registered MISR image data. Remote Sens. Environ. 2007, 107, 22-32. [CrossRef]

49. Horváth, Á. Improvements to MISR stereo motion vectors. J. Geophys. Res. Atmos. 2013, 118, 5600-5620. [CrossRef]

50. Hastings, D.; Dunbar, P. Global Land One-kilometer Base Elevation (GLOBE) Digital Elevation Model, Documentation. NGDC Key to Geophysical Records Documentation; 1999; Volume 1, p. 34. Available online: https://www.ngdc.noaa.gov/mgg/topo/report/globedocumentationmanual.pdf (accessed on 22 November 2018).

51. Lemoine, F.; Kenyon, S.; Factor, J.; Trimmer, R.; Pavlis, N.; Chinn, D.; Cox, C.; Klosko, S.; Luthcke, S.; Torrence, M.; et al. The Development of the Joint NASA GSFC and the National Imagery and Mapping Agency (NIMA) Geopotential Model EGM96. NASA/TP-1998-206861; 1998. Available online: https:/ / science.gsfc.nasa.gov/sed/ content/uploadFiles/publication_files/EGM96_NASA-TP-1998-206861.pdf (accessed on 22 November 2018).

52. Moroney, C.; Horvath, A.; Davies, R. Use of stereo-matching to coregister multiangle data from MISR. IEEE Trans. Geosci. Remote Sens. 2002, 40, 1541-1546. [CrossRef]

53. Lonitz, K.; Horváth, Á. Comparison of MISR and Meteosat-9 cloud-motion vectors. J. Geophys. Res. Atmos. 2011, 116. [CrossRef]

54. Gelaro, R.; McCarty, W.; Suárez, M.J.; Todling, R.; Molod, A.; Takacs, L.; Randles, C.A.; Darmenov, A.; Bosilovich, M.G.; Reichle, R.; et al. The Modern-Era Retrospective Analysis for Research and Applications, Version 2 (MERRA-2). J. Clim. 2017, 30, 5419-5454. [CrossRef]

55. Baker, N.; Pauley, P.; Langland, R.; Mueller, K.; Wu, D. An Assessment of the Impact of the Assimilation of NASA Terra MISR Atmospheric Motion Vectors on the NRL Global Atmospheric Prediction System. 2014. Available online: https://ams.confex.com/ams/94Annual/webprogram/Paper231106.html (accessed on 22 November 2018).

56. Luo, J.Z.; Jeyaratnam, J.; Iwasaki, S.; Takahashi, H.; Anderson, R. Convective vertical velocity and cloud internal vertical structure: An A-Train perspective. Geophys. Res. Lett. 2014, 41, 723-729. [CrossRef]

57. Marchand, R.; Ackerman, T.; Smyth, M.; Rossow, W.B. A review of cloud top height and optical depth histograms from MISR, ISCCP, and MODIS. J. Geophys. Res. 2010, 115, D16206. [CrossRef]

58. Liu, Q.; Weng, F. Advanced Doubling-Adding Method for Radiative Transfer in Planetary Atmospheres. J. Atmos. Sci. 2006, 63, 3459-3465. [CrossRef]

59. Rienecker, M.M.; Suarez, M.J.; Gelaro, R.; Todling, R.; Julio, B.; Liu, E.; Bosilovich, M.G.; Schubert, S.D.; Takacs, L.; Kim, G.-K.; et al. MERRA: NASA's Modern-Era Retrospective Analysis for Research and Applications. J. Clim. 2011, 24, 3624-3648. [CrossRef]

60. Gong, J.; Wu, D.L.; Limpasuvan, V. Meridionally tilted ice cloud structures in the tropical upper troposphere as seen by CloudSat. Atmos. Chem. Phys. 2015, 15, 6271-6281. [CrossRef]

61. Várnai, T.; Davies, R. Effects of Cloud Heterogeneities on Shortwave Radiation: Comparison of Cloud-Top Variability and Internal Heterogeneity. J. Atmos. Sci. 1999, 56, 4206-4224. [CrossRef]

62. Muller, J.-P. WINDS: Demonstrating How to Map the Earth's Winds $24 \mathrm{~h}$ Per Day-A Proposal to EE-8. Mullard Space Science Laboratory, University College London, London, UK. 2010. Available online: https:/ / cdn.knmi.nl/system/data_center_publications / files/000/068/580/original/winds_proposal_for_ ee8_issue_1.pdf?1495621279 (accessed on 22 November 2018).

63. Wood, R.; Wu, D.L.; Diner, D.J.; Garay, M.J.; Jovanovic, V. Spaceborne Atmospheric Boundary Layer Explorer (SABLE)-Earth Venture-2 Mission Proposal. University of Washington, Washington, USA. 2011. Available online: http:/ / cimss.ssec.wisc.edu/iwwg/iww11/talks/Session6_Diner.pdf (accessed on 22 November 2018).

64. Muller, J.-P.; Walton, D.; Fisher, D.; Cole, R. SMVs (Stereo Motion Vectors) from ASTR2-AATSR and MISRlite (Multi-Angle Infrared Stereo Radiometer) Constellation. In Proceedings of the 10th International Winds Workshop, Tokyo, Japan, 22-26 February 2010.

65. Rennó, N.O.; Williams, E.; Rosenfeld, D.; Fischer, D.G.; Fischer, J.; Kremic, T.; Agrawal, A.; Andreae, M.O.; Bierbaum, R.; Blakeslee, R.; et al. CHASER: An Innovative Satellite Mission Concept to Measure the Effects of Aerosols on Clouds and Climate. Bull. Am. Meteorol. Soc. 2013, 94, 685-694. [CrossRef]

66. Goldberg, A.C.; Kelly, M.A.; Boldt, J.; Wu, D.L.; Heidinger, A.; Wilson, J.P.; Ryan, K.J.; Morgan, M.F.; Yee, J.H.; Greenberg, J.M.; et al. Compact midwave imaging system (CMIS) for weather satellite applications. In Proceedings of the SPIE Defense + Security, Baltimore, MD, USA, 14-18 April 2018; p. 16. 
67. Hirschmuller, H. Stereo Processing by Semiglobal Matching and Mutual Information. IEEE Trans. Pattern Analysis Mach. Intell. 2008, 30, 328-341. [CrossRef] [PubMed]

68. Petrick, D.; Geist, A.; Albaijes, D.; Davis, M.; Sparacino, P.; Crum, G.; Ripley, R.; Boblitt, J.; Flatley, T. SpaceCube v2.0 space flight hybrid reconfigurable data processing system. In Proceedings of the 2014 IEEE Aerospace Conference, Big Sky, MT, USA, 1-8 March 2014; pp. 1-20.

69. Szeliski, R. Computer Vision; Springer: London, UK, 2011.

(C) (1)

(C) 2018 by the authors. Licensee MDPI, Basel, Switzerland. This article is an open access article distributed under the terms and conditions of the Creative Commons Attribution (CC BY) license (http:/ / creativecommons.org/licenses/by/4.0/). 